\title{
Recent star formation in the inner Galactic bulge seen by ISOGAL $\star$
}

\section{The central molecular zone}

\author{
K. Immer ${ }^{1,2}$, F. Schuller ${ }^{1}$, A. Omont ${ }^{3}$, and K. M. Menten ${ }^{1}$ \\ 1 Max-Planck-Institut für Radioastronomie, Auf dem Hügel 69, 53121 Bonn, Germany \\ e-mail: kimmer@mpifr-bonn.mpg.de \\ 2 Harvard-Smithsonian Center for Astrophysics, 60 Garden Street, 02138 Cambridge, MA, USA \\ 3 Institut d'Astrophysique de Paris, CNRS, 98bis Bd Arago, 75014 Paris, France
}

Received 9 August 2011 / Accepted 8 November 2011

\begin{abstract}
We present 5-38 $\mu \mathrm{m}$ spectroscopic observations of a sample of 68 ISOGAL sources with unknown natures, taken with the Spitzer Infrared Spectrograph. Based on the characteristics and the slope of their spectra, we classified the sources as young or late-type evolved objects. These sources were selected to test selection criteria based on the ISOGAL [7]-[15] color and the spatial extent parameter $\sigma_{15}$. We revised these criteria until they reliably distinguished between young and late-type evolved objects and then applied them to all ISOGAL sources in the central molecular zone (CMZ), resulting in the selection of 485 sources believed to be young. Furthermore, we added 656 Midcourse Space Experiment (MSX) sources to the CMZ sample that fulfilled $F_{\mathrm{E}} / F_{\mathrm{D}}>2$ with $F_{\mathrm{D}}$ and $F_{\mathrm{E}}$ being the flux densities in the $D(15 \mu \mathrm{m})$ and $E(21 \mu \mathrm{m})$ bands. After obtaining $\frac{L_{\mathrm{bol}}}{F_{15}}$ conversion factors, we calculated the bolometric luminosity, $L_{\mathrm{bol}}$, values for the CMZ sample and subsequently the masses of the sources. Applying a Kroupa initial mass function, we derived the total mass in young objects that have been formed over the last $1 \mathrm{Myr}$, resulting in an average star formation rate of 0.08 solar masses per year for the CMZ.
\end{abstract}

Key words. stars: formation - infrared: stars - Galaxy: stellar content - H II regions - Galaxy: center

\section{Introduction}

The central few hundred parsecs of the Milky Way present an exceptional environment in our Galaxy. This region produces around $5-10 \%$ of the infrared luminosity of the Galaxy and contains ca. $10 \%$ of its neutral gas $\left(\sim 5-10 \times 10^{7} M_{\odot}\right)($ e.g. Güsten 1989; Rodríguez-Fernández et al. 2004). The conditions in this central molecular zone $\left(\mathrm{CMZ}\right.$, the inner $\pm 1.5^{\circ} \times \pm 0.5^{\circ}$ around the Galactic center) are very extreme, the temperature, the velocity dispersion, the pressure, and the estimated magnetic field strengths are much higher than in the Galactic disk (Morris \& Serabyn 1996). From a statistical point of view, a study of the star formation activity in the CMZ can uncover if star formation in this region is enhanced or suppressed by these extreme conditions.

The CMZ harbors many sites of massive star formation. The prominent emission of giant molecular clouds from infrared to radio wavelengths indicates recent bursts of star formation near the Galactic center. However, most star formation studies in the CMZ are focused on the most massive and highly active star clusters near Sgr $\mathrm{A}^{*}$ or the giant $\mathrm{H}_{\text {II }}$ complexes Sgr B2 and Sgr C.

Thus, to obtain an extensive overview of the star formation activity in the CMZ, a large number of young stellar objects (YSOs) have to be identified in this region. One method that is applicable to a large number of sources to identify YSO candidates is to use color selection criteria. However, to be reliable,

* Figure 3 is available in electronic form at http://www . aanda.org photometric color criteria always require a spectroscopic confirmation of the nature of objects in a well-selected sample.

At a particular time in its evolution a YSO begins to heat its dust in the surrounding birth cloud, which then re-emits this absorbed energy at infrared wavelengths. Studying massive star formation at infrared wavelengths (preferably with spectroscopic methods) therefore gives insight into the characteristics of the circumstellar dust, as well as the properties of the embedded star.

Large surveys combining several bands in the near- and midinfrared are important tools in systematic studies of young stellar populations. In this publication, we use data from the ISOGAL (Omont et al. 2003) and the Midcourse Space Experiment (MSX, Price et al. 2001) surveys. The ISOGAL survey comprises observations of the inner Galaxy at 7 and $15 \mu \mathrm{m}$, taken with the infrared camera ISOCAM onboard the Infrared Space Observatory (ISO) with a spatial resolution better than 6". The MSX project is a multiwavelength infrared survey of the entire Galactic plane in four spectral bands at $8,12,15$, and $21 \mu \mathrm{m}$ with a spatial resolution of $\sim 18^{\prime \prime}$.

Schuller et al. (2006) (hereafter: Paper I) defined selection criteria based on the [7]-[15] ISOGAL color (where [7] and [15] are the magnitudes at 7 and $15 \mu \mathrm{m}$, respectively, measured with ISO), as well as the magnitude uncertainty at $15 \mu \mathrm{m}, \sigma_{15}$ (residual between the fitted point spread function and the actual source profile, see Schuller et al. 2003), which serves as a parameter for the spatial extension of the source (explained below). These criteria were established from a group of point sources from the ISOGAL point source catalog 
(Schuller et al. 2003; Omont et al. 2003) with known natures, dividing them into young objects ${ }^{1}$ (YOs) and late-type evolved objects (LEOs). Since YOs are embedded in a dust cocoon, they appear more extended at $15 \mu \mathrm{m}$ than LEOs. As explained in Paper I, a high value of $\sigma_{15}$ indicates that the source is marginally resolved at $15 \mu \mathrm{m}$. To be present in the PSC, such sources still have angular diameters $\lesssim 30^{\prime \prime}$, but values of $10^{\prime \prime}-20^{\prime \prime}$ (FWHM) are typical.

In this publication, these selection criteria are tested on a sample of sources with unknown evolutionary stages. We determine the nature of these sources from the characteristics and the slope of their midinfrared spectra, which were obtained with the Spitzer Infrared Spectrograph (Houck et al. 2004). By means of this sample of unknown sources we refine the selection criteria until they reliably distinguish between YOs and LEOs.

The final goal is to apply a high-quality version of the ISO selection criteria on all ISOGAL sources in the CMZ in order to find YOs and to characterize the star formation activity near the center of our Galaxy.

This paper is divided into two parts. The first part comprises the analysis of the infrared spectra. In Sects. 2, 2.1, and 2.2 we present the selection of sources for our test sample, the Spitzer observations of the infrared spectra, and the data processing of the spectra. In the next section, the classification of the studied sources is described. In the last section of this part, we give refined selection criteria, which will then be applied to all ISOGAL sources in the CMZ in the second part of this publication in order to find YOs within the inner $3^{\circ}$ of our Galaxy. In Sect. 3.2 we derive the bolometric luminosities, as well as the masses of all sources in our CMZ sample and finally determine the average star formation rate in the CMZ.

\section{Verification of the ISO color selection criteria}

Infrared colors are a powerful tool for distinguishing between various classes of objects. Based on fluxes at 12, 25, and $60 \mu \mathrm{m}$ measured with the InfraRed Astronomy Satellite (IRAS), Wood \& Churchwell (1989) defined criteria to identify ultracompact H II (UCH II) regions based on far infrared colors. In the 1990s, midinfrared surveys conducted with MSX and ISO covered the Galactic plane with much higher spatial resolution and sensitivity than IRAS. Lumsden et al. (2002) defines color criteria based on MSX and the near infrared 2MASS survey to select candidate massive YSOs. Similarly, from identifications of ISOGAL point sources with known objects, it has been found that massive YSOs and UCH II regions show [7]-[15] colors above 2 mag (Felli et al. 2002). Moreover, most sources with [15] $<4$ mag and [7]-[15] $>2$ mag have MSX counterparts with flux ratios $F_{21 \mu \mathrm{m}} / F_{15 \mu \mathrm{m}}$ above 2 , making their interpretation as YSO very likely, since YSOs are characterized by strong rising continuum emission in the infrared range. In addition, in Paper I it has been shown that the spatial extent at $15 \mu \mathrm{m}$, as it appears in the ISOGAL catalog, can help in determining whether sources brighter than $300 \mathrm{mJy}$ at $15 \mu \mathrm{m}$ (or [15] $\leq 4.5 \mathrm{mag}$ ) are YOs or LEOs. Infrared sources with ISOGAL characteristics similar to those of known massive YOs, but without any known counterpart, are thus good massive YSO candidates.

Not all of the sources in the ISOGAL catalog have been detected at both of the covered wavelengths ( 7 and $15 \mu \mathrm{m})$. Since

\footnotetext{
1 In this publication, the term "young objects" refers to YSOs (a phase in the star formation process before the ionization of the surrounding birth cloud) and $\mathrm{H}_{\text {II }}$ regions (a later phase where the star starts to ionize its dust cocoon).
}

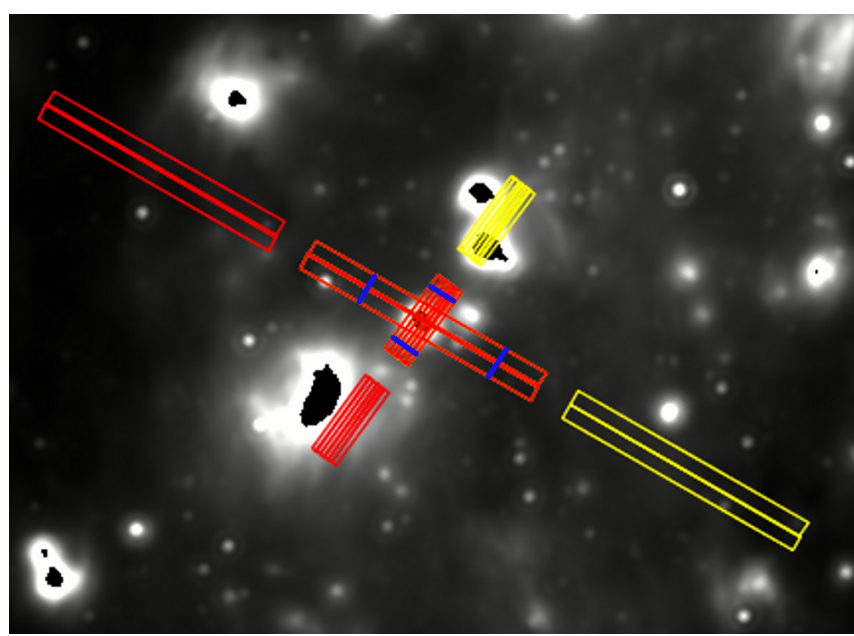

Fig. 1. Footprint of the IRS slits shown on a $24 \mu \mathrm{m}$ image around J174505.6-291018. In that case, the map consists of 6 pointings with the SL module, and 2 pointings with the LL module. Positions in the slits where background spectra were obtained are shown in blue.

sources brighter than 8 mag at $7 \mu \mathrm{m}$ should have been detected by the ISOGAL survey, a lower limit for the [7] magnitude of $8 \mathrm{mag}$ was assigned to sources that were not detected at $7 \mu \mathrm{m}$.

Glass et al. (2001) published a catalog of long-period variable stars, which are located near the Galactic center and appear as bright ISOGAL sources. In the $0.1 \mathrm{deg}^{2}$ region of the ISOGAL survey overlapped by the Glass et al. (2001) observations, 95 sources brighter than $5.25 \mathrm{mag}$ at $15 \mu \mathrm{m}$ show [7]-[15] colors above 1.8 mag. Sixteen of these sources can be matched with long-period variable stars from the Glass et al. (2001) catalog, and were removed from our sample. After rejecting another six sources associated with known OH/IR stars and five showing near infrared spectra typical of AGB stars (Schultheis et al. 2003), we finally selected 68 ISOGAL sources as targets for follow-up spectroscopic observations with Spitzer. This test sample is complete down to a $15 \mu \mathrm{m}$ flux density of $140 \mathrm{mJy}$ over a $0.1 \mathrm{sq}$. deg. area.

Despite our effort to remove known LEOs from our test sample, we realized later that two of our targets (J174602.8-290359, J174623.0-285845) have been identified as OH/IR stars by Sjouwerman et al. (1998). These sources have been helpful in establishing the identification criteria for LEOs in our test sample.

\subsection{Observation and Inspection of the data}

The spectroscopic data were obtained in March 2005 with the two low-resolution modules of the Infrared Spectrograph (IRS; Houck et al. 2004), onboard the Spitzer Space Telescope (Werner et al. 2004). The 68 sources were observed in the IRS-mapping mode, with the low-resolution modules SL (Short-wavelength, Low-resolution) and LL (Long-wavelength, Low-resolution). Both modules were subdivided into three submodules that cover different parts of the two wavelength ranges (SL2: 5-7.5 $\mu \mathrm{m}$, SL3: 7.3-8.7 $\mu \mathrm{m}$, SL1: 7.5-15 $\mu \mathrm{m}$, LL2: 14-21 $\mu \mathrm{m}$, LL3: 19.4 $21.7 \mu \mathrm{m}$, LL1: $21-40 \mu \mathrm{m})$. Each map consisted of between three and 17 pointings with the SL module, and between one and four pointing(s) with the LL module, depending on the size of the source as seen in the ISOGAL image. As an example, Fig. 1 shows a footprint of the IRS slits on a Spitzer/MIPS $24 \mu \mathrm{m}$ image around the source J174505.6-291018. The ramp duration was set to $6 \mathrm{~s}$ for each module, and two cycles were required per 
ramp. In most cases, this was sufficient to achieve signal-to-noise ratios of 100 or more over the entire wavelength range.

We began with a first inspection of the data. Depending on the detection of the ISOGAL sources at short $(5-15 \mu \mathrm{m})$ or long (15-40 $\mu \mathrm{m})$ midinfrared wavelengths in the spectroscopic data, we divided the sources into different groups:

- A) ISOGAL sources, detected as one source in the SL, as well as in the LL data at the same position (49 sources);

- B) ISOGAL sources, only detected in the LL data (8 sources);

- C) ISOGAL sources, detected neither in the SL nor in the LL data (4 sources);

- D) Confusion of several Spitzer sources in the SL or LL data at the position of the ISOGAL source (7 sources).

Only the sources in groups A and B were processed further, giving a total of 57 sources. Since the group C ISOGAL sources were not detected with Spitzer at higher resolution, these sources are probably false detections in the ISOGAL catalog.

\subsection{Data reduction}

The data reduction was conducted with the Spitzer software IRSCLEAN $^{2}$ (to clean rogue pixels in the data) and $\mathrm{SPICE}^{3}$ (to extract the spectra). The spectra were calibrated by SPICE with calibration files that are automatically selected by the software (for more information about the calibration see the SPICE User's Guide $^{4}$ ).

A direct background subtraction cannot be conducted with SPICE. Therefore, the source and the background spectra were determined separately, and the background subtraction was conducted later. Since the infrared background in the Galactic center region varies greatly on short spatial scales, the background spectra were obtained from the same subslit as the source spectra. Several background spectra were extracted from different positions and pointings around the source (the number of obtained background spectra varies from source to source, depending on the infrared source density around the target sources). The blue lines in Fig. 1 show the positions where background spectra were obtained around J174505.6-291018. To obtain a mean background spectrum at the position of the source, the weighted average of the background spectra and the associated errors were determined.

Per subslit and submodule (SL2, SL3, SL1, LL2, LL3, LL1), the source spectra were extracted from those pointings where the emission of the source could be distinguished from the background emission and the emission of other infrared sources. Per pointing, the source spectra of the two cycles were averaged and the mean background spectrum subtracted. Per submodule, all background-subtracted source spectra were added together. Then, the background subtracted source spectra of the six submodules were assembled to produce the final source spectrum.

Seventeen sources of the test sample show flux jumps between the SL and the LL module spectra around $14 \mu \mathrm{m}$. These are due to extended emission that falls in the slits in combination with the different slit widths of the two modules covering areas of different sizes around the sources.

\footnotetext{
${ }^{2}$ http://ssc.spitzer.caltech.edu/archanaly/ contributed/irsclean/IRSCLEAN_MASK.html

${ }^{3}$ http://ssc.spitzer.caltech.edu/dataanalysistools/ tools/spice/

${ }^{4}$ http://ssc.spitzer.caltech.edu/postbcd/doc/spice. pdf
}

Twentyseven of the 57 test sample sources were only detected at $15 \mu \mathrm{m}$ in ISOGAL, and a lower limit of 8 mag was assigned to their [7] magnitudes. Therefore, their [7]-[15] colors are also only lower limits. Furthermore, it should be mentioned that the ISOGAL magnitudes at 7 and $15 \mu \mathrm{m}$ were not observed at the same time for $63 \%$ of the 57 sources (sources with right ascension, RA < 17:45:40). Due to possible variability of the sources, this could lead to errors in the [7]-[15] parameter.

\subsection{Spectroscopic properties of the test sample}

The most common features in the IRS wavelength range, detected in our sources, are silicate absorption bands, emission features from polycyclic aromatic hydrocarbons (PAHs), and finestructure lines from ionized species. A broad silicate absorption band at around $9.8 \mu \mathrm{m}$ is present in all the spectra, but the central wavelength changes marginally from source to source. Further silicate absorption features are detected at 18, 22.3, and/or $23 \mu \mathrm{m}$ (e.g. Molster et al. 2002). These silicate features are indicative of O-rich envelopes with high mass infall rates.

In a few sources, absorption bands at around 5.95, 6.7, and $7.4 \mu \mathrm{m}$ seem to be present. According to Gerakines et al. (1995), the absorption at $6 \mu \mathrm{m}$ is partly due to a bending mode of water ice. The $6.7 \mu \mathrm{m}$ absorption band can be partly explained by absorption through methanol ice (Quirico et al. 2000), whereas the band at $7.4 \mu \mathrm{m}$ is possibly caused by acetaldehyde $\left(\mathrm{CH}_{3} \mathrm{HCO}\right)$ ice (Schutte et al. 1998).

Emission from PAHs requires a strong ultraviolet field to be excited and thus is frequently found at the edges of $\mathrm{H}$ in regions. Eleven of the sources show unambiguous emission of PAHs at one or more of the following wavelengths: $6.2 \mu \mathrm{m}, 7.7 \mu \mathrm{m}$, $8.6 \mu \mathrm{m}, 11.3 \mu \mathrm{m}$, and $16.4 \mu \mathrm{m}$. However, due to confusion of the ice absorption bands at wavelengths shorter than $8 \mu \mathrm{m}$, the $\mathrm{PAH}$ emission features in this wavelength range could not be unambiguously identified in several sources.

In our source spectra, the detection of PAH emission bands is always seen in combination with at least one of the following forbidden fine-structure lines: [Ar II] $(6.97 \mu \mathrm{m}),[\mathrm{Ne}$ II] $(12.83 \mu \mathrm{m})$, and $\left[\mathrm{S}_{\mathrm{III}}\right](18.7 \mu \mathrm{m})$. The most frequent emission line, $[\mathrm{Ne}$ II], is detected in the direction of 21 sources. In five of the sources the detection is weak and could be spurious. The [Ar II] and the [S III] lines are observed in the direction of seven and fourteen sources, respectively. Although the occurrence of these emission lines often indicates the existence of $\mathrm{H}$ in regions and therefore the presence of hot massive stars, this emission can also arise from the warm, ionized, interstellar medium surrounding the sources. This fact complicates the background subtraction. The detection of the emission lines should therefore be considered with caution; nevertheless, the lines can still be used as a diagnostic tool.

In several sources, the $S(1)$ and $S(3)$ rotational emission lines of $\mathrm{H}_{2}$ at $17.03 \mu \mathrm{m}$ and $9.66 \mu \mathrm{m}$ (within the broad silicate absorption feature) are detected. They are tracers of warm molecular gas (e.g. Kaufman et al. 2006; Fiolet et al. 2010).

Table 1 shows the spectral features that were identified in the source spectra. The first column contains the source names. Columns 2-5 list the detection of PAH emission features, as well as forbidden fine structure lines ([Ar II $],[\mathrm{Ne} I I],\left[\mathrm{S}_{\mathrm{III}}\right]$ ). Where the identification of a feature was not clear, question marks were set in the table. Columns 6-8 mark the detection of emission of the $\mathrm{S}(1)$ and $\mathrm{S}(3)$ rotational lines of $\mathrm{H}_{2}$ at $17.03 \mu \mathrm{m}$ and $9.66 \mu \mathrm{m}$, as well as silicate absorption at 22.3 or $23 \mu \mathrm{m}$. In the last column, the detection of ice absorption bands at around 5.95, 6.7, or $7.4 \mu \mathrm{m}$ are mentioned. 
Table 1. Detected spectral features of the sources.

\begin{tabular}{|c|c|c|c|c|c|c|c|c|}
\hline \multirow[t]{2}{*}{ Source name } & \multirow{2}{*}{$\begin{array}{c}\text { PAH } \\
\text { Emission }\end{array}$} & \multirow{2}{*}{$\begin{array}{c}{[\mathrm{Ar} \mathrm{II}]} \\
6.97 \mu \mathrm{m}\end{array}$} & \multirow{2}{*}{$\begin{array}{c}{[\mathrm{Ne} \text { II }]} \\
12.83 \mu \mathrm{m}\end{array}$} & \multirow{2}{*}{$\begin{array}{c}{\left[\mathrm{S}_{\mathrm{III}}\right]} \\
18.7 \mu \mathrm{m}\end{array}$} & \multicolumn{2}{|c|}{$\mathrm{H}_{2}$} & \multirow{2}{*}{$\begin{array}{c}\text { Absorption } \\
22.3 \text { and/or } 23 \mu \mathrm{m}\end{array}$} & \multirow[t]{2}{*}{ Ice absorption bands } \\
\hline & & & & & $\mathrm{S}(1)(17.03 \mu \mathrm{m})$ & $\mathrm{S}(3)(9.66 \mu \mathrm{m})$ & & \\
\hline J174457.4-291003 & & & & & $\checkmark ?$ & & & $\checkmark ?$ \\
\hline J174459.1-290653 & & & & & & & $\checkmark$ & \\
\hline J174500.7-291007 & $\checkmark ?$ & $\checkmark ?$ & $\checkmark ?$ & & & $\checkmark$ & & \\
\hline J174501.0-285622 & & & & $\checkmark$ & $\checkmark$ & & $\checkmark$ & \\
\hline J174504.9-291146 & & $\checkmark$ & $\checkmark$ & $\checkmark ?$ & & $\checkmark$ & & \\
\hline J174505.1-290937 & $\checkmark ?$ & $\checkmark ?$ & & & $\checkmark$ & & $\checkmark$ & \\
\hline J174505.6-291018 & $\checkmark$ & $\checkmark$ & $\checkmark$ & $\checkmark$ & & & & $\checkmark ?$ \\
\hline J174506.5-291118 & & & $\checkmark$ & $\checkmark$ & & & & \\
\hline J174508.0-290655 & & & & & & & $\checkmark$ & \\
\hline J174508.0-291039 & & $\checkmark ?$ & & $\checkmark$ & & & & \\
\hline J174508.1-290840 & $\checkmark$ & & $\checkmark$ & $\checkmark$ & $\checkmark ?$ & & $\checkmark ?$ & $\checkmark ?$ \\
\hline J174508.7-290348 & $\checkmark$ & $\checkmark ?$ & & & & $\checkmark$ & & \\
\hline J174511.3-290621 & $\checkmark$ & $\checkmark ?$ & $\checkmark ?$ & & & & & \\
\hline J174512.6-290646 & $\checkmark ?$ & & & & & & $\checkmark$ & $\checkmark ?$ \\
\hline J174515.4-291213 & & & & & & & & \\
\hline J174515.9-290155 & $\checkmark ?$ & $\checkmark ?$ & & & & & $\checkmark ?$ & $\checkmark ?$ \\
\hline J174516.2-290315 & & & & & & & & $\checkmark ?$ \\
\hline J174517.1-291155 & & $\checkmark ?$ & & & & & $\checkmark ?$ & \\
\hline J174517.8-290813 & & & & & & & $\checkmark$ & \\
\hline J174518.1-290439 & & & $\checkmark$ & $\checkmark$ & & & & \\
\hline J174518.1-291051 & $\checkmark ?$ & $\checkmark$ & $\checkmark$ & $\checkmark$ & & & & \\
\hline J174518.3-290628 & & & & & $\checkmark ?$ & & $\checkmark$ & $\checkmark ?$ \\
\hline J174520.0-290750 & & & & & $\checkmark$ & & $\checkmark$ & \\
\hline J174520.1-290638 & & & & & & & & \\
\hline J174520.7-290213 & & & $\checkmark$ & $\checkmark$ & & & & \\
\hline J174522.1-291059 & $\checkmark ?$ & $\checkmark ?$ & $\checkmark$ & $\checkmark ?$ & & $\checkmark ?$ & & \\
\hline J174522.4-290242 & $\checkmark$ & $\checkmark ?$ & $\checkmark ?$ & & $\checkmark ?$ & & $\checkmark$ & \\
\hline J174523.3-290331 & & & & & & $\checkmark$ & $\checkmark ?$ & \\
\hline J174523.5-290225 & $\checkmark$ & & $\checkmark$ & & & & $\checkmark$ & \\
\hline J174523.9-290310 & & & & & & & & \\
\hline J174524.8-290529 & & & & & $\checkmark$ & & & \\
\hline J174524.9-290318 & & $\checkmark ?$ & $\checkmark$ & & & & & \\
\hline J174525.7-290942 & $\checkmark$ & $\checkmark$ & $\checkmark$ & $\checkmark$ & $\checkmark$ & & & \\
\hline J174527.2-291126 & & & & & & & $\checkmark ?$ & \\
\hline J174527.4-290655 & & & & & $\checkmark$ & & $\checkmark ?$ & \\
\hline J174529.5-291021 & $\checkmark ?$ & $\checkmark$ & $\checkmark$ & & & & & $\checkmark ?$ \\
\hline J174553.3-290406 & & & & $\checkmark ?$ & & & & \\
\hline J174555.6-290521 & $\checkmark ?$ & & $\checkmark ?$ & & & $\checkmark ?$ & & \\
\hline J174557.7-290302 & $\checkmark ?$ & & & & & $\checkmark ?$ & $\checkmark$ & \\
\hline J174600.1-290150 & & & $\checkmark$ & $\checkmark$ & & & $\checkmark$ & \\
\hline J174602.5-290427 & & & & & $\checkmark ?$ & & & \\
\hline J174602.8-290359 & & & & & & & & \\
\hline J174612.7-285958 & & & $\checkmark$ & $\checkmark$ & & & $\checkmark$ & \\
\hline J174613.1-290327 & $\checkmark ?$ & & & & & & $\checkmark$ & \\
\hline J174613.5-290024 & $\checkmark$ & & & $\checkmark$ & & & $\checkmark$ & \\
\hline J174617.6-285857 & $\checkmark ?$ & & & & & & $\checkmark$ & \\
\hline J174618.0-290245 & $\checkmark$ & & $\checkmark ?$ & $\checkmark ?$ & & $\checkmark ?$ & $\checkmark$ & \\
\hline J174618.2-290136 & $\checkmark ?$ & & & & & & & \\
\hline J174619.4-285611 & & & & & & & $\checkmark$ & \\
\hline J174620.8-290532 & & & & & & & $\checkmark$ & \\
\hline J174623.0-285845 & & & & & & & & \\
\hline $\mathrm{J} 174623.8-285300$ & & & & & & & & \\
\hline J174624.9-290345 & & & & & & & $\checkmark$ & \\
\hline J174631.1-290652 & $\checkmark ?$ & & $\checkmark$ & & & & $\checkmark$ & \\
\hline J174631.2-285448 & & & & $\checkmark ?$ & & & $\checkmark$ & \\
\hline J174631.8-285027 & $\checkmark$ & & & $\checkmark$ & & & $\checkmark$ & \\
\hline J174632.9-285115 & $\checkmark$ & $\checkmark$ & $\checkmark$ & $\checkmark$ & & & $\checkmark$ & \\
\hline
\end{tabular}




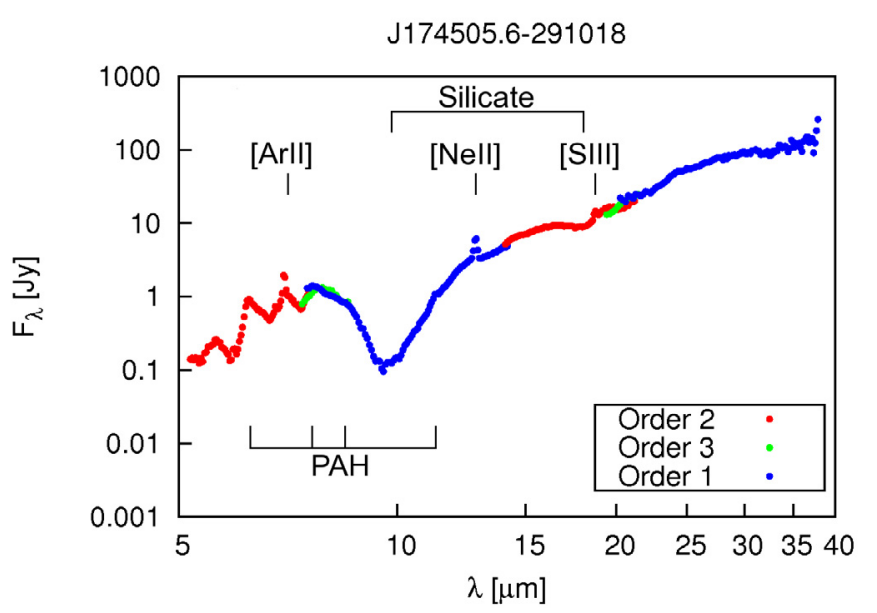

Fig. 2. Background-subtracted spectrum of the source J174505.6-291018. The data of the different subslits are presented by the three different colors (green: SL2 or LL2; blue: SL3 or LL3; red: SL1 or LL1). Denoted are the silicate absorption features, the PAH emission bands, and the forbidden fine structure lines [Ar II], [Ne II], and [S III].

As an example, Fig. 2 shows the background-subtracted spectrum of the source J174505.6-291018. The three different colors represent the data of the different subslits (green: SL2 or LL2; blue: SL3 or LL3; red: SL1 or LL1). The spectrum shows emission lines of [Ar II], [Ne II], and [S III], as well as two silicate absorption features at 9.8 and $18 \mu \mathrm{m}$. Furthermore, the detected $\mathrm{PAH}$ emission features are indicated. The spectra of the remaining 56 sources are shown in Fig. 3.

\subsubsection{Classification of the 57 sources}

The vast majority of ISOGAL sources brighter than 5 mag at $15 \mu \mathrm{m}$ are either of young stellar nature or are LEOs (Paper I). While YOs are characterized by very steep rising continua in the infrared range, LEOs show more flat to falling continua. An inspection of our spectra shows that some of the sources seem to be composed of a falling SL and a rising LL spectrum.

The two sources that were identified as $\mathrm{OH} / \mathrm{IR}$ stars by Sjouwerman et al. (1998) both show flat spectra. The only detectable spectral features are the silicate absorption bands at 9.8 and $18 \mu \mathrm{m}$. Neither PAH emission bands nor forbidden fine structure lines are visible in the spectra.

To identify the sources as YOs or LEOs, we looked at both the slope of the spectrum and the detected spectral features. To allow for a quantitative classification of the spectral slopes, we determined the ratio of the flux densities at 33 and $5.3 \mu \mathrm{m}, \frac{F_{33} \mu \mathrm{m}}{F_{5.3} \mathrm{~mm}}$. The fluxes at these two wavelengths were selected in order to obtain the slope over a large part of the spectrum (both SL and LL parts) and to avoid spectral features at the short wavelengths end and the large flux jumps between data points at the long wavelength end. We expect high values for this parameter for YOs with a steep rising spectrum and lower values for more evolved sources with a flat spectrum. The spectra of sources that show a flux jump between the SL and the LL module were first corrected for the mismatch, and then the slope parameter was determined for these sources. The two OH/IR stars in our test sample have slope parameter values of two and three. After an inspection of the data, we set the threshold for the identification of a source as a YO to be $\frac{F_{33 \mu \mathrm{m}}}{F_{5.3 \mu \mathrm{m}}} \geq 10$. Sources that were only detected with the
SL1 and the LL modules or only the LL modules were identified by comparing their spectra to the other sources.

Depending on the value of the slope parameter, we classified the sources as YOs or LEOs. If possible, we subdivided the group of YOs into YSOs or HII regions depending on the detection of forbidden fine structure lines. In the spectra of YSOs or LEOs we do not expect the detection of PAH emission bands or forbidden fine structure lines. In fourteen of the sources, the identification of the spectral features is not clear. However, the slope of their spectra classifies them as YOs.

- YO (49 sources)

- YSO (14 sources)

- Steep rising spectrum

- No PAH features or forbidden fine structure lines

- H II region (Н II) (21 sources)

- Steep rising spectrum

- Forbidden fine structure lines

- Steep rising spectrum but no other clear features (14 sources)

- LEO (8 sources)

- Flat spectrum

- No PAH features or forbidden fine structure lines.

Furthermore, it should be mentioned that the classification in YSOs and $\mathrm{H}$ II regions is not completely reliable. On one hand, a young $\mathrm{H}$ iI region does not have to show emission of forbidden fine structure lines yet. On the other hand, a YSO could have forbidden fine-structure emission lines in its spectrum, originating in the ambient medium or a nearby source. However, the slope of the spectrum unambiguously identifies these sources as YOs.

Following Paper I, the flux ratio $F_{\mathrm{E}} / F_{\mathrm{D}}$ of an MSX point source in the $\mathrm{E}(21 \mu \mathrm{m})$ and $\mathrm{D}(15 \mu \mathrm{m})$ bands can be used as an identification criterion for young massive stars, if $F_{\mathrm{E}} / F_{\mathrm{D}}>2$. For 21 of our ISOGAL sources, we could find an MSX point source within 8 ". (Only MSX sources with flux quality flags of 2-4 were considered.) Based on their spectra, eighteen of them were identified as YSO or $\mathrm{H}$ II regions and their MSX counterparts showed $F_{\mathrm{E}} / F_{\mathrm{D}}$ flux ratios above two. The three other sources were classified as LEOs, supported by the flux ratios of their MSX counterparts $F_{\mathrm{E}} / F_{\mathrm{D}}$ of $0.85,1.31$, and 1.57 .

In addition, we looked for radio emission in the vicinity of our ISOGAL sources in images of the VLA archive at 1.4, 4.7, and $8.4 \mathrm{GHz}$ in order to identify potential $\mathrm{H}$ II regions. At the positions of six ISOGAL sources, which were classified as $\mathrm{H}$ II regions on the basis of their spectra, radio emission was detected. Several other sources are located at the edge of diffuse radio emission.

Table 2 shows the ISOGAL colors and the spectral identification of the sources. Columns 1-5 list the sources names, together with their ISOGAL magnitudes at 7 and $15 \mu \mathrm{m}$, the [7]-[15] color, and the spatial extent parameter $\sigma_{15}$. The sixth column contains the slope parameter $\frac{F_{33} \mu \mathrm{m}}{F_{5.3} \mu \mathrm{m}}$. In the last two columns the identification of the sources and the detection of radio emission at the positions of the sources in the VLA images are reported.

\subsection{Test of the ISOGAL selection criteria}

One of the intentions of this publication has been to determine if the applied photometric color criteria ([15] $<5.25 \mathrm{mag}$, [7]-[15] > $1.8 \mathrm{mag}$ ) permit the selection of only YOs in a sample of ISOGAL point sources. The identification of eight LEOs in our test sample shows that the color criteria do not constrain the group of sources to only objects with young stellar nature. We 
A\&A 537, A121 (2012)

Table 2. ISOGAL colors, spatial extent and slope parameters, as well as the identification of the sources.

\begin{tabular}{|c|c|c|c|c|c|c|c|}
\hline Source name & $\begin{array}{c}{[7]} \\
(\mathrm{mag})\end{array}$ & $\begin{array}{c}{[15]} \\
\text { (mag) }\end{array}$ & {$[7]-[15]$} & $\begin{array}{c}\sigma_{15} \\
(\mathrm{mag})\end{array}$ & $F_{33 \mu \mathrm{m}} / F_{5.3 \mu \mathrm{m}}$ & Identification & Radio source? \\
\hline J174457.4-291003 & 8 & 4.95 & 3.05 & 0.1 & & YSO & - \\
\hline J174459.1-290653 & 6.89 & 3.49 & 3.4 & 0.05 & 138 & YSO & - \\
\hline J174500.7-291007 & 8 & 4.92 & 3.08 & 0.03 & 250 & YO & - \\
\hline J174501.0-285622 & 8 & 4.83 & 3.17 & 0.19 & & $\mathrm{H}_{\text {II }}$ & - \\
\hline J174504.9-291146 & 4.27 & 1.97 & 2.3 & 0.11 & 58 & $\mathrm{H}_{\text {II }}$ & $\checkmark$ \\
\hline J174505.1-290937 & 8 & 3.83 & 4.17 & 0.11 & 48 & YO & - \\
\hline J174505.6-291018 & 5.24 & 1.78 & 3.46 & 0.09 & 678 & $\mathrm{H}_{\text {II }}$ & $\checkmark$ \\
\hline J174506.5-291118 & 5.29 & 0.88 & 4.41 & 0.07 & 782 & $\mathrm{H}_{\text {II }}$ & $\checkmark$ \\
\hline J174508.0-290655 & 8 & 4.34 & 3.66 & 0.14 & & YSO & - \\
\hline J174508.0-291039 & 6.59 & 4.24 & 2.35 & 0.11 & 63 & $\mathrm{H}_{\text {II }}$ & - \\
\hline J174508.1-290840 & 7.69 & 5.11 & 2.58 & 0.06 & 240 & $\mathrm{H}_{\text {II }}$ & $\checkmark$ \\
\hline J174508.7-290348 & 8 & 4.17 & 3.83 & 0.12 & 230 & $\mathrm{H}_{\text {II }}$ & - \\
\hline J174511.3-290621 & 6.44 & 4.31 & 2.13 & 0.07 & 72 & YO & - \\
\hline J174512.6-290646 & 8 & 4.97 & 3.03 & 0.03 & 70 & YO & - \\
\hline J174515.4-291213 & 8 & 3.4 & 4.6 & 0.11 & 1242 & YSO & - \\
\hline J174515.9-290155 & 8 & 2.63 & 5.37 & 0.13 & 246 & YSO & - \\
\hline J174516.2-290315 & 5.66 & 1.03 & 4.63 & 0.19 & 163 & YSO & - \\
\hline J174517.1-291155 & 8 & 5.02 & 2.98 & 0.09 & & YSO & - \\
\hline J174517.8-290813 & 7.3 & 4.1 & 3.2 & 0.09 & 290 & $\mathrm{YO}$ & - \\
\hline J174518.1-290439 & 6.3 & 2.54 & 3.76 & 0.05 & 139 & $\mathrm{H}_{\text {II }}$ & $\checkmark$ \\
\hline J174518.1-291051 & 6.51 & 3 & 3.51 & 0.08 & 2285 & $\mathrm{H}_{\text {II }}$ & $\checkmark$ \\
\hline J174518.3-290628 & 8 & 5.18 & 2.82 & 0.1 & 22 & YSO & - \\
\hline J174520.0-290750 & 7.34 & 5.09 & 2.25 & 0.08 & & YSO & - \\
\hline J174520.1-290638 & 8 & 4.93 & 3.07 & 0.12 & & YO & - \\
\hline J174520.7-290213 & 4.57 & 0.4 & 4.17 & 0.12 & 554 & YO & - \\
\hline J174522.1-291059 & 6.81 & 4.61 & 2.2 & 0.04 & 124 & $\mathrm{H}_{\text {II }}$ & - \\
\hline J174522.4-290242 & 8 & 3.68 & 4.32 & 0.17 & 109 & $\mathrm{H}_{\text {II }}$ & - \\
\hline J174523.3-290331 & 5.52 & 2.29 & 3.23 & 0.14 & 24 & YO & - \\
\hline J174523.5-290225 & 8 & 4.62 & 3.38 & 0.14 & & YO & - \\
\hline J174523.9-290310 & 6.09 & 3.29 & 2.8 & 0.14 & 10 & YO & - \\
\hline J174524.8-290529 & 8 & 3.89 & 4.11 & 0.19 & & YSO & - \\
\hline J174524.9-290318 & 8 & 3.33 & 4.67 & 0.14 & 386 & $\mathrm{H}_{\text {II }}$ & - \\
\hline J174525.7-290942 & 8 & 4.68 & 3.32 & 0.15 & 73 & $\mathrm{H}_{\text {II }}$ & - \\
\hline J174527.2-291126 & 7.54 & 5.15 & 2.39 & 0.1 & 73 & YSO & - \\
\hline J174527.4-290655 & 8 & 5.12 & 2.88 & 0.13 & & YSO & - \\
\hline J174529.5-291021 & 6.89 & 4.51 & 2.38 & 0.07 & 271 & $\mathrm{H}_{\text {II }}$ & - \\
\hline J174553.3-290406 & 8 & 3.55 & 4.45 & 0.09 & & $\mathrm{YO}$ & - \\
\hline J174555.6-290521 & 7.05 & 3.77 & 3.28 & 0.09 & & $\mathrm{H}_{\text {II }}$ & - \\
\hline J174557.7-290302 & 7.53 & 5.05 & 2.48 & 0.06 & 11 & YO & - \\
\hline J174600.1-290150 & 8 & 3.48 & 4.52 & 0.06 & & $\mathrm{H}_{\text {II }}$ & - \\
\hline J174602.5-290427 & 8 & 3.81 & 4.19 & 0.19 & & YSO & - \\
\hline J174602.8-290359 & 5.9 & 2.43 & 3.47 & 0.08 & 2 & LEO & - \\
\hline J174612.7-285958 & 7.42 & 5.08 & 2.34 & 0.14 & 71 & $\mathrm{H}_{\text {II }}$ & - \\
\hline J174613.1-290327 & 8 & 4.75 & 3.25 & 0.13 & 2 & LEO & - \\
\hline J174613.5-290024 & 6.84 & 4.52 & 2.32 & 0.22 & & $\mathrm{H}_{\text {II }}$ & - \\
\hline J174617.6-285857 & 8 & 4.81 & 3.19 & 0.13 & 1 & LEO & - \\
\hline J174618.0-290245 & 8 & 5.24 & 2.76 & 0.21 & 186 & $\mathrm{H}_{\text {II }}$ & - \\
\hline J174618.2-290136 & 6.35 & 0.88 & 5.47 & 0.19 & 62 & YSO & - \\
\hline J174619.4-285611 & 6.98 & 4.57 & 2.41 & 0.19 & 6 & LEO & - \\
\hline J174620.8-290532 & 6.13 & 4.22 & 1.91 & 0.04 & 1 & LEO & - \\
\hline J174623.0-285845 & 5.07 & 2.97 & 2.1 & 0.05 & 3 & LEO & - \\
\hline $\mathrm{J} 174623.8-285300$ & 5.21 & 3.2 & 2.01 & 0.03 & 6 & LEO & - \\
\hline J174624.9-290345 & 8 & 5.23 & 2.77 & 0.09 & 1 & LEO & - \\
\hline J174631.1-290652 & 8 & 5.14 & 2.86 & 0.09 & 47 & YO & - \\
\hline J174631.2-285448 & 6.9 & 4.76 & 2.14 & 0.13 & 17 & YO & - \\
\hline J174631.8-285027 & 8 & 4.12 & 3.88 & 0.21 & & $\mathrm{H}_{\text {II }}$ & - \\
\hline J174632.9-285115 & 7.67 & 5.21 & 2.46 & 0.14 & 219 & $\mathrm{H}_{\text {II }}$ & - \\
\hline
\end{tabular}




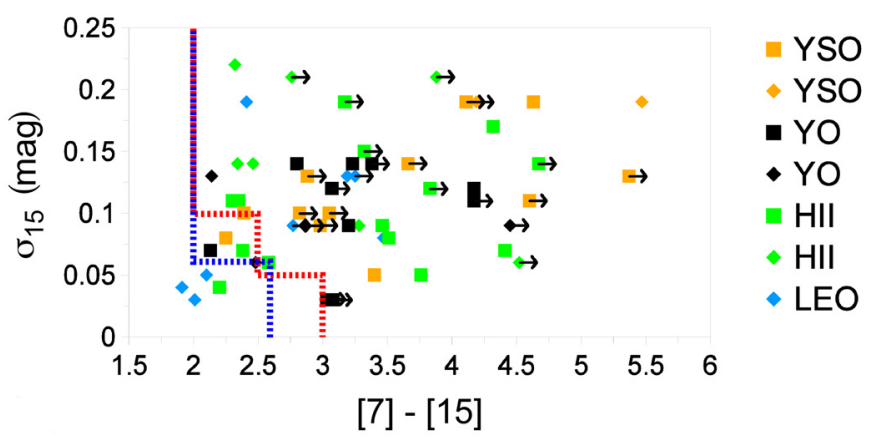

Fig. 4. The image shows the spatial extension parameter $\sigma_{15}$ plotted against the [7]-[15] color. The arrows indicate lower limits of the [7][15] colors for the sources that were only detected at $15 \mu \mathrm{m}$. The squares mark the sources whose [7] and [15] colors were not observed at the same time, diamonds highlight sources that were simultaneously observed at 7 and $15 \mu \mathrm{m}$. The red line represents the selection criteria of Paper I, the blue line the selection criteria defined in this publication. The image shows that color selection criteria that are combined with the spatial extension parameter $\sigma_{15}$ permit the distinction between young and evolved objects with a low contamination rate of LEOs.

confirm that a combination of the spatial extension of the sources at $15 \mu \mathrm{m}$, characterized by the parameter $\sigma_{15}$, with their [7]-[15] colors gives a reliable method of distinguishing between YOs and LEOs.

Figure 4 plots $\sigma_{15}$ against the [7]-[15] colors. The image shows that YOs and LEOs cannot be divided into two separate sections in the plot. However, criteria based on the ISOGAL colors, as well as the spatial extension parameter, allow the extraction of YOs from a mixed sample with a small contamination by evolved sources. Applying the selection criteria

$2 \leq[7]-[15]<2.5$ and $\sigma_{15}>0.1 \mathrm{mag}$

$2.5 \leq[7]-[15]<3$ and $\sigma_{15}>0.05 \mathrm{mag}$

[7]-[15] $\geq 3$, no constraint on $\sigma_{15}$,

which were defined in Paper I from a group of ISOGAL sources with known evolutionary states, to our test sample results in the selection of 44 YOs and five LEOs, corresponding to a LEO contamination rate of $10.2 \%$ (5/49). To reduce the LEO contamination rate by selecting more YOs and to simplify the selection criteria, we refined the criteria to

\section{$2 \leq[7]-[15]<2.6$ and $\sigma_{15} \geq 0.06 \mathrm{mag}$}

\section{$2.6 \leq[7]-[15]$, no constraint on $\sigma_{15}$.}

These criteria would yield the same number of LEOs but 48 YOs, resulting in a LEO contamination rate of $9.4 \%(5 / 53)$. These criteria also agree with the ISOGAL source sample of Paper I and will be used in the second part of this publication in order to identify YOs in the CMZ in the ISOGAL point source catalog.

To estimate the final LEO contamination rate, the remaining 11 sources for which no spectrum was assembled and the 27 sources that were identified beforehand as late evolved stars also have to be considered. If we apply the defined selection criteria on the 27 rejected sources, nine of them are classified as YOs, increasing the contamination rate to $22.6 \%$ (14/62). All 11 unidentified sources of our test sample of 68 sources are sorted in the group of YOs. The sources labeled as group C sources are probably false detections in the ISOGAL catalog, increasing the contamination rate to $27.3 \%(18 / 66)$. Only one of the group D objects has an MSX counterpart within $8^{\prime \prime}$, which has a flux density ratio of $F_{\mathrm{E}} / F_{\mathrm{D}}>2$. The ISOGAL sources in group D are resolved in more than one source at short wavelengths by the Spitzer observations. The ISOGAL colors of these sources can be dominated by either young sources or more evolved objects in the cluster. The contamination rate is therefore $34.2 \%$ $(25 / 73)$ if the group D objects are defined as more evolved objects and contribute to the contamination rate or $24.7 \%(18 / 73)$ if they are identified as YOs. In conclusion, the contamination rate with non-YOs is in the range 25 to $35 \%$.

Another spectroscopic study of YSO candidates in the CMZ was recently conducted by An et al. (2011). Based on IRAC colors, they selected 107 point sources for their sample and obtained high-resolution Spitzer/IRS spectra for these targets. The shape of the absorption profile of $\mathrm{CO}_{2}$ ice at $15 \mu \mathrm{m}$ served as their identification criterion. In total, they identified 35 YSOs and possible YSOs.

Since we only have low-resolution data for our sources, we could not test the An et al. identification criterion on our test sample. A comparison of the An et al. source sample and our test sample showed that both have three sources in common. An et al. identify only one of them as a possible YSO (source $\mathrm{J} 174529.5-291021$ ) on the basis of the $\mathrm{CO}_{2}$ ice absorption profile. Beside this ice absorption band, our Spitzer/IRS spectrum of this source also shows emission lines of [ArII] and [NeII] in its spectrum. Thus, we identified this source as an $\mathrm{H}$ II region. We conclude that this source could be a transition object between YSOs and $\mathrm{H}_{\text {II }}$ regions, showing signatures of both phases. We also identified the other two sources as Hir regions. An et al. exclude these sources being YSOs.

While the An et al. study focuses only on YSOs, we obtain a broader picture of the star formation history in the CMZ since we also include objects in our test sample that are in later evolutionary stages of the star formation process, such as $\mathrm{H}$ in regions.

\section{Star formation in the central molecular zone}

In this part, we apply the defined selection criteria on all ISOGAL sources that have been detected in the central molecular zone in order to determine the star formation activity in the central $450 \mathrm{pc}$ of our Galaxy. YO candidates are selected in an area covering $\pm 1.5^{\circ} \times \pm 0.5^{\circ}$ around the Galactic center. The list of ISOGAL sources in the CMZ is compared with the MSX point source catalog (PSC) and MSX sources with a flux density ratio of $F_{\mathrm{E}} / F_{\mathrm{D}} \geq 2$ without an ISOGAL counterpart within $8^{\prime \prime}$ are added to the $\mathrm{CMZ}$ source list of YO candidates.

We derived bolometric luminosities for all sources in the CMZ sample from their flux densities at $15 \mu \mathrm{m}$ by using $\frac{L_{\mathrm{bol}}}{F_{15}}$ conversion factors that are obtained from our test sample sources. Furthermore, we determine mass estimates for all CMZ sample sources to determine the total mass in YOs that has been formed over the past $\sim 1 \mathrm{Myr}$ and thus derive an average star formation rate for the $\mathrm{CMZ}$.

\subsection{Selection of the candidate young objects}

\subsubsection{ISOGAL point sources}

The data processing of the ISOGAL survey (Omont et al. 2003) and the creation of the ISOGAL-DENIS point source catalog have been extensively described in Schuller et al. (2003). Here we only use ISOGAL fields that have been observed at 7 and 
Table 3. Characteristics of the ISOGAL fields used in the present analysis.

\begin{tabular}{lllcc}
\hline \hline Name & \multicolumn{2}{c}{ Filters } & FOV & Pixel \\
\hline FC-00121-00003 & LW5 & LW9 & $9^{\prime} \times 17^{\prime}$ & $6^{\prime \prime}$ \\
FC-00112-00035 & LW6 & LW9 & $28^{\prime} \times 20^{\prime}$ & $6^{\prime \prime}$ \\
FC-00109+00031 & LW6 & LW9 & $34^{\prime} \times 23^{\prime}$ & $6^{\prime \prime}$ \\
FC-00090-00003 & LW5 & LW9 & $21^{\prime} \times 17^{\prime}$ & $6^{\prime \prime}$ \\
FC-00062-00006 & LW5 & LW9 & $12^{\prime} \times 17^{\prime}$ & $3^{\prime \prime}$ \\
FC-00062-00040 & LW6 & LW9 & $15^{\prime} \times 12^{\prime}$ & $6^{\prime \prime}$ \\
FC-00039+00018 & LW6 & LW9 & $39^{\prime} \times 9^{\prime}$ & $6^{\prime \prime}$ \\
FC-00027-00006 & LW5 & LW9 & $19^{\prime} \times 17^{\prime}$ & $3^{\prime \prime}$ \\
FC+00004+00040 & LW6 & LW9 & $46^{\prime} \times 14^{\prime}$ & $6^{\prime \prime}$ \\
FC+00005-00024 & LW5 & LW9 & $18^{\prime} \times 15^{\prime}$ & $6^{\prime \prime}$ \\
FC+00034-00005 & LW5 & LW9 & $14^{\prime} \times 16^{\prime}$ & $3^{\prime \prime}$ \\
FC+00037+00017 & LW5 & LW9 & $34^{\prime} \times 10^{\prime}$ & $6^{\prime \prime}$ \\
FC+00059+00002 & LW5 & LW9 & $14^{\prime} \times 7^{\prime}$ & $3^{\prime \prime}$ \\
FC+00062-00014 & LW5 & LW9 & $13^{\prime} \times 6^{\prime}$ & $3^{\prime \prime}$ \\
FC+00066-00041 & LW6 & LW9 & $46^{\prime} \times 12^{\prime}$ & $6^{\prime \prime}$ \\
FC+00067+00038 & LW6 & LW9 & $19^{\prime} \times 14^{\prime}$ & $6^{\prime \prime}$ \\
FC+00089-00009 & LW6 & LW9 & $18^{\prime} \times 20^{\prime}$ & $6^{\prime \prime}$ \\
FC+00124-00032 & LW6 & LW9 & $23^{\prime} \times 22^{\prime}$ & $6^{\prime \prime}$ \\
FC+00127+00035 & LW6 & LW9 & $23^{\prime} \times 20^{\prime}$ & $6^{\prime \prime}$ \\
\hline
\end{tabular}

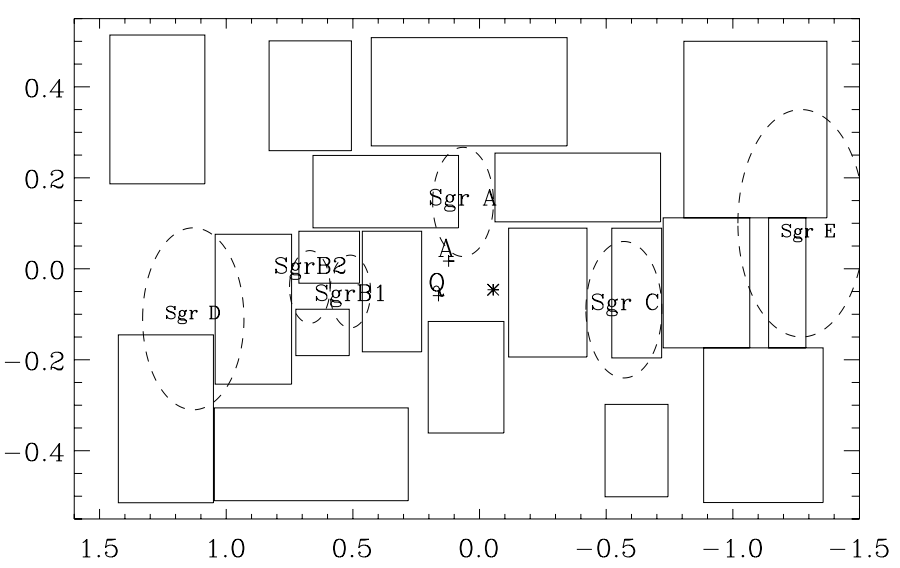

Fig. 5. Boundaries of the ISOGAL fields used for the present study (see also Table 3), in Galactic coordinates. Some well known regions are indicated: Sgr A* (asterisk), the Arches cluster (A), the Quintuplet cluster (Q), as well as the Sgr A, Sgr B1, Sgr B2, Sgr C, Sgr D, and Sgr E star-forming regions.

$15 \mu \mathrm{m}$. A list of the fields (Col. 1) with which the sources were observed, the filter numbers (Cols. 2, 3), field of view of the fields (Col. 4), and the pixel scales of the fields (Col. 5) are given in Table 3.

The ISOGAL observations were designed to exclude strong IRAS sources, with $F_{12 \mu \mathrm{m}} \geq 6 \mathrm{Jy}$ in general, to avoid saturation effects of the detector. However, for most fields observed around the Galactic center, narrow filters and $3^{\prime \prime}$ pixels were used to allow the observations of brighter sources (up to $F_{12 \mu \mathrm{m}} \sim 20 \mathrm{Jy}$ ); nevertheless, the Galactic center itself and a couple of other brightest star-forming regions could not be imaged. Figure 5 shows the boundaries of the ISOGAL fields, listed in Table 3. In addition, known star-forming regions are indicated.

As shown in the first part of this publication, as well as in Paper I, source samples based only on color selection criteria are contaminated with some post-main sequence stars (planetary nebulae, OH/IR stars with extreme mass losses), although most of the sources are associated with massive stars. Combining a color selection criterion with an indication of spatial extension (i.e., removing the point-like sources) allows infrared sources to be selected that are very likely massive (young) objects.

The application of the selection criteria defined in the first part of this publication to the ISOGAL fields listed in Table 3 leads to selecting 485 ISOGAL sources with [15] $<5.25 \mathrm{mag}$. As described in Sects. 2 and 2.2, a lower limit for the [7] magnitude was assigned to sources that were not detected at $7 \mu \mathrm{m}$.

\subsubsection{MSX point sources}

The Midcourse Space Experiment performed a survey of the Galactic plane at four midinfrared wavelengths: 8, 12, 15, and $21 \mu \mathrm{m}$, with a pixel scale of $18^{\prime \prime}$. The sensitivity of the MSX catalog version 2.3 (Egan et al. 2003) is on the order of $50 \mathrm{mJy}$ at $8 \mu \mathrm{m}$ and $1-2 \mathrm{Jy}$ at 15 and $21 \mu \mathrm{m}$.

As shown in Paper I, the longest wavelengths of MSX provide another color criterion $\left(F_{\mathrm{E}} / F_{\mathrm{D}} \geq 2\right)$ for selecting probable young massive objects, with little contamination by LEOs and planetary nebulae (see also Lumsden et al. 2002). To have reliable flux density values for the MSX sources at 15 and $21 \mu \mathrm{m}$, we only considered sources with flux quality flags of 2-4 in the $D$ and $E$ bands. Besides the ISOGAL sources, we selected MSX point sources in the CMZ without an ISOGAL counterpart within $8^{\prime \prime}$ that fulfilled the specified MSX color criterion, resulting in $656 \mathrm{MSX}$ sources.

In total, our CMZ sample contains 1141 YO candidates. Figure 6 shows the distribution of the CMZ source sample in Galactic longitude $l$ and Galactic latitude $b$. Most of the MSX sources are located at $0^{\circ} \leq l \leq 0.5^{\circ}$, which could not be observed by ISOGAL because of detector saturation. Most of the sources in the CMZ sample are confined to the Galactic plane $\left(|b| \leq 0.2^{\circ}\right)$.

\subsection{Discussion}

Among the 485 ISOGAL sources, 190 (almost 40\%) have an MSX counterpart within a search radius of $8^{\prime \prime}$. The distribution of ISO-MSX position separations (Fig. 7) does not show any increase with larger separations, as would be expected from random associations. In addition, the agreement between ISO and MSX magnitudes is generally good. Thus, we do not expect a large contribution by false associations in our CMZ sample. However, the MSX magnitudes tend to be lower (the sources appear brighter) than the ISO sources, especially at $7 / 8 \mu \mathrm{m}$. This can be partly explained by different central wavelengths $(8 \mu \mathrm{m}$ vs. $7 \mu \mathrm{m}$ ) that can make a difference for these red objects. Some part of the observed difference may also be due to a Malmquist bias: with a sensitivity more limited for MSX than ISOGAL, the brighter MSX sources will tend to be associated. In addition, the lower spatial resolution of MSX than for ISO can also introduce a bias in that direction, when a single MSX source is resolved into several ISOGAL point sources.

Figure 8 shows the positions of the 1833 MSX sources in the CMZ, detected in all four MSX bands, within the [15]-[21] vs. [8]-[15] color-color diagram. Almost half of these sources appear in a "clump", around [8]-[15] 2 and [15]-[21] 0.5 . Only $\sim 20$ of our ISO-MSX sources are located in this clump and could actually be "normal" field objects. On the contrary, $80 \%$ of the ISO-MSX objects have an $F_{\mathrm{E}} / F_{\mathrm{D}}$ ratio above two. Therefore, we conclude that our candidate young massive objects, selected on the basis of their ISO magnitudes, show peculiar MSX colors and indeed trace a specific population. 


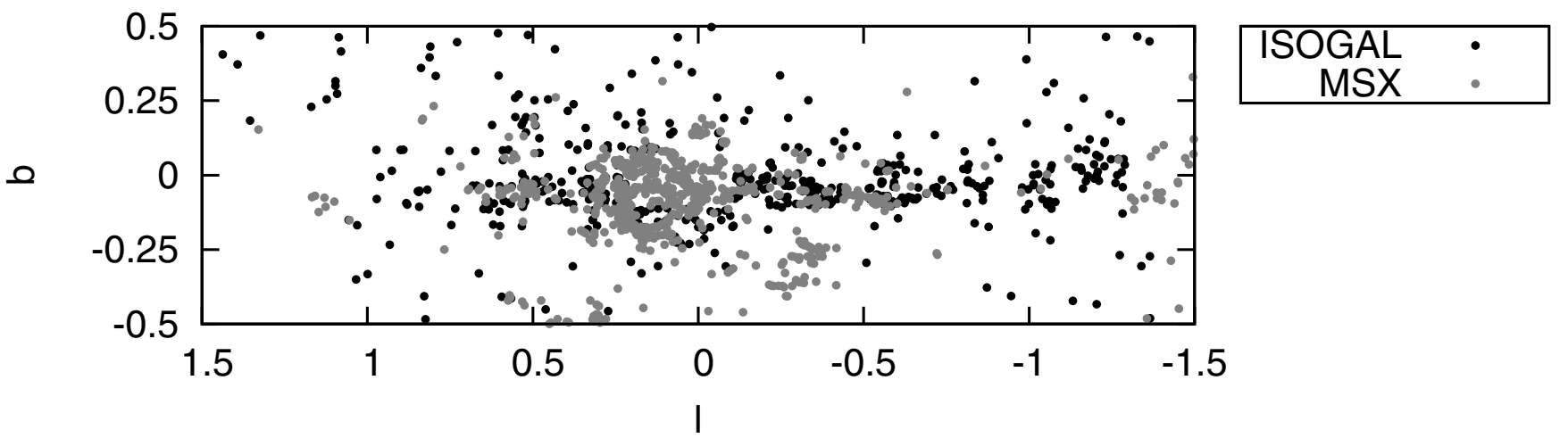

Fig. 6. Areal coverage of our CMZ sample with Galactic longitude $l$ and Galactic latitude $b$. Black dots mark the ISOGAL sources, gray dots the MSX sources without ISOGAL counterparts.
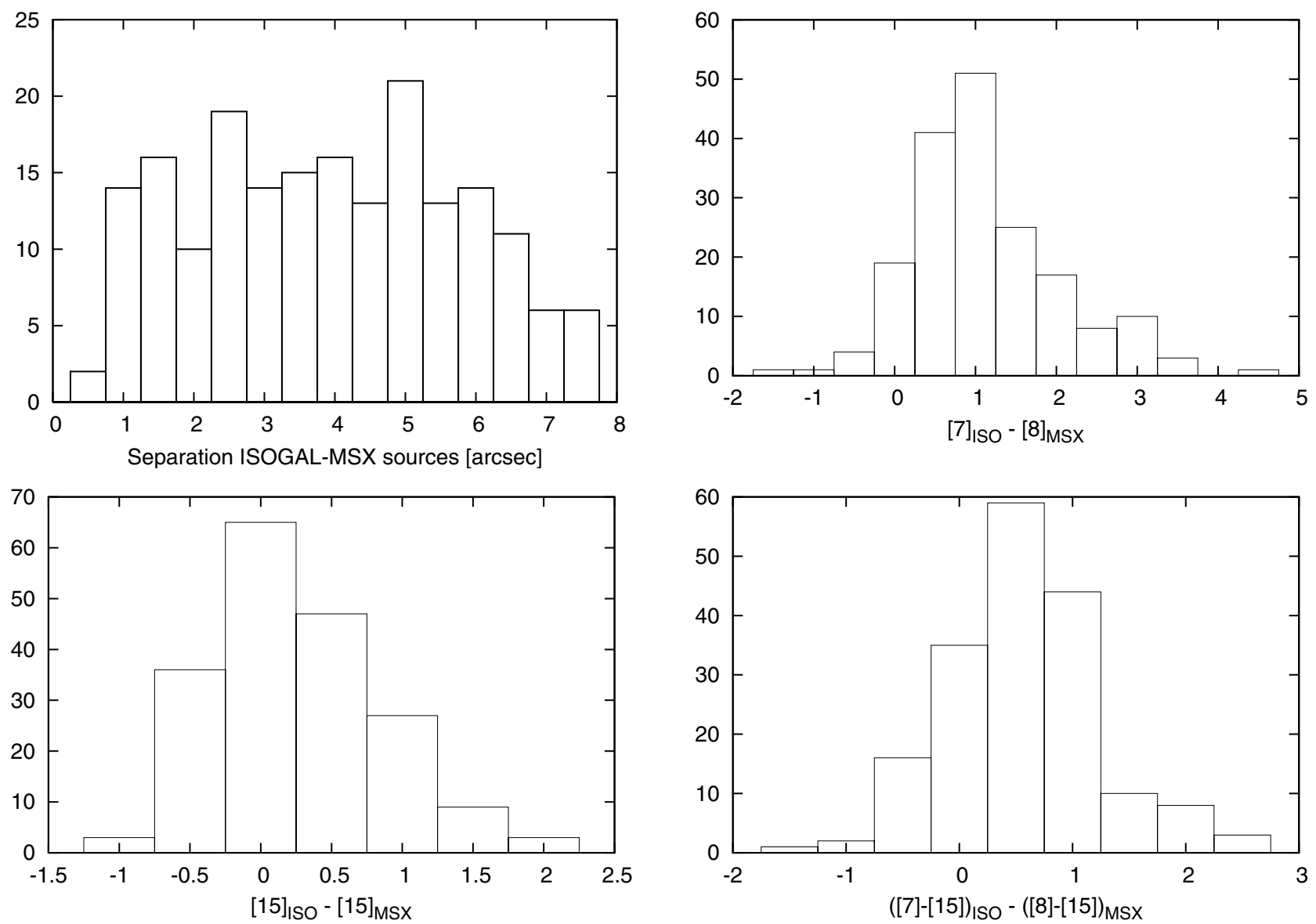

Fig. 7. From top left to bottom right, distributions of: angular separation between the ISOGAL and the associated MSX source, ISO-MSX $7 \mu \mathrm{m}$ magnitude differences, ISO-MSX $15 \mu$ m magnitude differences, and ISO-MSX differences in colors.

\subsubsection{Interstellar extinction}

Interstellar extinction in the direction of the CMZ can exceed 30 mag in the $V$ band. Since we want to estimate the bolometric luminosities of our sources from their flux densities at $15 \mu \mathrm{m}$, we have to correct them for interstellar reddening for all sources in the CMZ sample. We used an extinction map, computed by Schultheis et al. (2009), which is based on the combination of photometric data from the 2MASS and IRAC/GLIMPSE surveys. We derived a value for $A_{\mathrm{V}}$ toward each object in our CMZ source list as an average of all data points within a radius of $0.5^{\prime}$ of the object position. For CMZ sources that are not located within the area of this extinction map, we used an older extinction map (Schultheis et al. 1999) that is based on photometric data from the DENIS survey.

Furthermore, we extracted $\frac{A_{15}}{A_{\mathrm{K}_{\mathrm{S}}}}$ conversion factors from the results of Jiang et al. (2005), who give a value for each ISOGAL field. For those MSX sources that are not located in an ISOGAL field, an average value of 0.4 was set as the $\frac{A_{15}}{A_{K_{S}}}$ conversion factor. The $\frac{A_{\mathrm{K}_{\mathrm{S}}}}{A_{\mathrm{V}}}$ conversion factor was obtained from the results of Nishiyama et al. (2008) $\left(\frac{A_{\mathrm{K}_{\mathrm{S}}}}{A_{\mathrm{V}}}=0.062\right)$. This value was derived specifically for the Galactic center environment. 


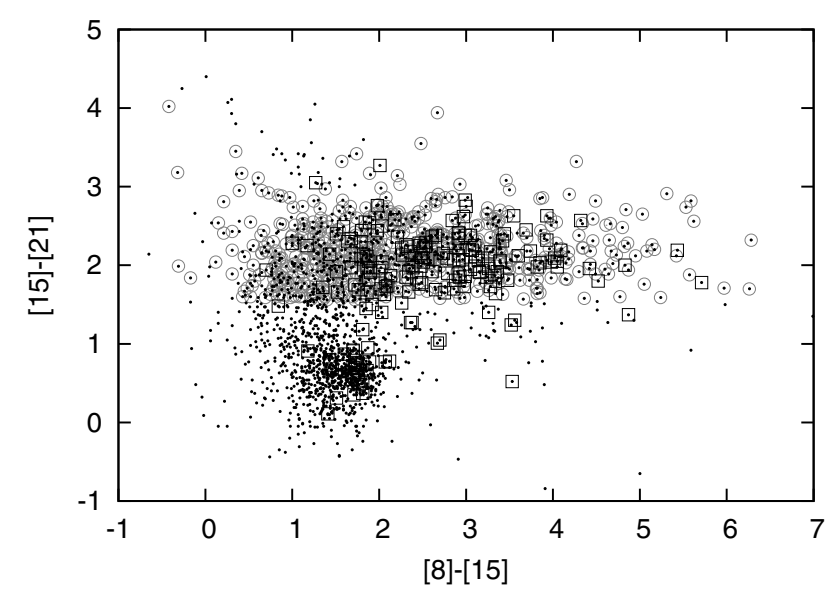

Fig. 8. MSX Color-color diagram. All MSX sources in the CMZ, detected in all four MSX bands, are plotted with black dots. The black boxes mark all MSX sources with an ISOGAL counterpart within $8^{\prime \prime}$ (177 sources). $656 \mathrm{MSX}$ sources with $F_{\mathrm{E}} / F_{\mathrm{D}} \geq 2$ without an ISOGAL counterpart within $8^{\prime \prime}$ are shown as gray circles. The MSX sources, marked with boxes and circles, have flux quality flags $\geq 1$ in the $A$ and $C$ bands and $\geq 2$ in the $D$ and $E$ bands.

The corrected magnitudes at $15 \mu \mathrm{m}$ of the ISOGAL sources were then converted into flux densities. The MSX flux density values were corrected by translating the flux density into magnitude values, subtracting $A_{15}$, and then retranslating the corrected magnitude values into flux densities.

\subsubsection{Bolometric luminosities}

To derive a conversion factor between the flux density $F_{15}$ and the luminosity $L_{\mathrm{bol}}$, we fitted the Spitzer/IRS spectra of the 49 YOs in our test sample with the SED fitting tool of Robitaille et al. (2007). This tool contains 20000 YSO models, covering a wavelength range of 0.01 to $5000 \mu \mathrm{m}$, calculated at ten different viewing angles and 50 circular apertures, which results in a total number of ten million SEDs (Robitaille et al. 2006). The models cover a wide stellar mass range of 0.1 to $50 M_{\odot}$ and all evolutionary stages from prestellar to pre-main sequence. During the fitting, the tool applies all the models to the entered data, leaving the distance and the external foreground extinction $A_{\mathrm{V}}$ as free parameters within a user specified range. The result is a list of 10000 fits that are characterized by a $\chi^{2}$ value. Since the parameter space is sampled too sparsely to allow an accurate determination of the $\chi^{2}$ surface minima, and hence the appropriate confidence intervals, a specified $\chi^{2}$ criterion for "good" fits has to be selected. We defined the following criterion: $\chi^{2}-\chi_{\text {best }}^{2} \leq 3 \cdot n_{\text {points }}$ (Robitaille et al. 2007) with $\chi_{\text {best }}^{2}$ the $\chi^{2}$ value of the best-fitting model and $n_{\text {points }}$ the number of data points used in the fitting process. Although this selection criterion is kind of arbitrary, a stricter criterion would imply an overinterpretation of the fitting results.

Since the distance and the external foreground extinction serve as free parameters, a distance range and a range for the visual extinction $A_{\mathrm{V}}$ have to be specified. We assumed that all $\mathrm{CMZ}$ sources are located at the Galactic center distance $(8.0 \mathrm{kpc}$, Reid et al. 2009). Accordingly, we chose the fitted distance range to be 7-9 kpc. We set $A_{\mathrm{V}}$ to be 15 to $50 \mathrm{mag}$, corresponding to typical maximal and minimal values of $A_{\mathrm{V}}$ in the area of the 49 sources, extracted from the extinction map of Schultheis et al. (2009).
For the fitting, we selected between six and eleven data points per spectrum, spread between 5 and $35 \mu \mathrm{m}$, to reflect the underlying continuum. Since the SED tool does not model emission lines, PAHs, or ice features, we avoided the selection of data points in thus affected regions of the spectrum. Since the fitting tool permits entering data taken with different apertures, we can select the data points from the spectrum without correcting for the flux jumps that are visible in some of the spectra. For the size of the apertures, we chose the widths of the SL and LL modules.

We selected the models following the above-defined criterion and calculated the bolometric flux densities using the luminosity values and the distance values of the different models. Then, we reconverted them into luminosity values, using a single distance of $8.0 \mathrm{kpc}$ for all models. We calculated the weighted average and standard deviation of the bolometric luminosities for each source with the inverse of the $\chi^{2}$ values as the weights.

In the next step, we plotted the obtained bolometric luminosities of the 49 ISOGAL sources against their corrected ISOGAL flux densities at $15 \mu \mathrm{m}$ (left hand image in Fig. 9). The standard deviations served as error bar values. A linear fit to the data provided the conversion factor $\left(\frac{L_{\mathrm{bol}}}{F_{15}}\right)_{\text {ISOGAL }}=(4447 \pm 106) L_{\odot} / \mathrm{Jy}$. For the 18 ISOGAL sources with an MSX counterpart within $8^{\prime \prime}$, we plotted their bolometric luminosities against the corrected MSX flux densities at $15 \mu \mathrm{m}$ (right image in Fig. 9). The slope of the fitted line is $\left(\frac{L_{\text {bol }}}{F_{15}}\right)_{\mathrm{MSX}}=(3736 \pm 209) L_{\odot} / \mathrm{Jy}$.

Afterwards, we calculated the bolometric luminosities for all sources in our CMZ sample from their $F_{15}$ flux densities. The luminosity values range from $0.5 \times 10^{3}$ to $\sim 10^{6} L_{\odot}$, with a total of $\sim 2.5 \times 10^{7} L_{\odot}$.

\subsubsection{Masses}

It is commonly accepted that hydrogen burning in high-mass stars sets in while the star is still accreting material from the circumstellar birth cloud. At this phase, the surrounding material is heated and eventually ionized by the UV radiation of the star. Based on our ISOGAL selection criteria, the sources in our CMZ sample are selected to have bright midinfrared emission from warm dust and hence are still highly embedded in their birth clouds but already contain a central heating source. We can therefore assume that most of the sources in our CMZ sample are likely to be intermediate- to high-mass stars that have already started hydrogen burning and are thus already on the zero age main sequence (ZAMS). Assuming that the bolometric luminosity of each source is that of a single ZAMS star, mass estimates for all CMZ sample sources have been derived, using the relation (e.g. Eddington 1926; Griffiths et al. 1988; Bhattacharya et al. 2008)

$$
\frac{L}{L_{\odot}} \approx\left(\frac{M}{M_{\odot}}\right)^{3.5} \text {. }
$$

The distribution of the masses, which ranges from 6 to $58 M_{\odot}$, is roughly consistent with a Salpeter or Kroupa initial mass function (IMF, Salpeter 1955; Kroupa 2001). However, the histogram (see Fig. 10) clearly shows that low-mass objects are missing from our CMZ sample because of the selection criterion [15] $<5.25 \mathrm{mag}$, corresponding to a lower mass limit of $6.3 M_{\odot}$. While most of the total luminosity in a young population comes from high-mass stars, most of the total mass is contained in the high number of low-mass stars. Since we are only selecting the most massive objects (the most luminous ones), we miss the stars with lower masses. To obtain a complete picture 

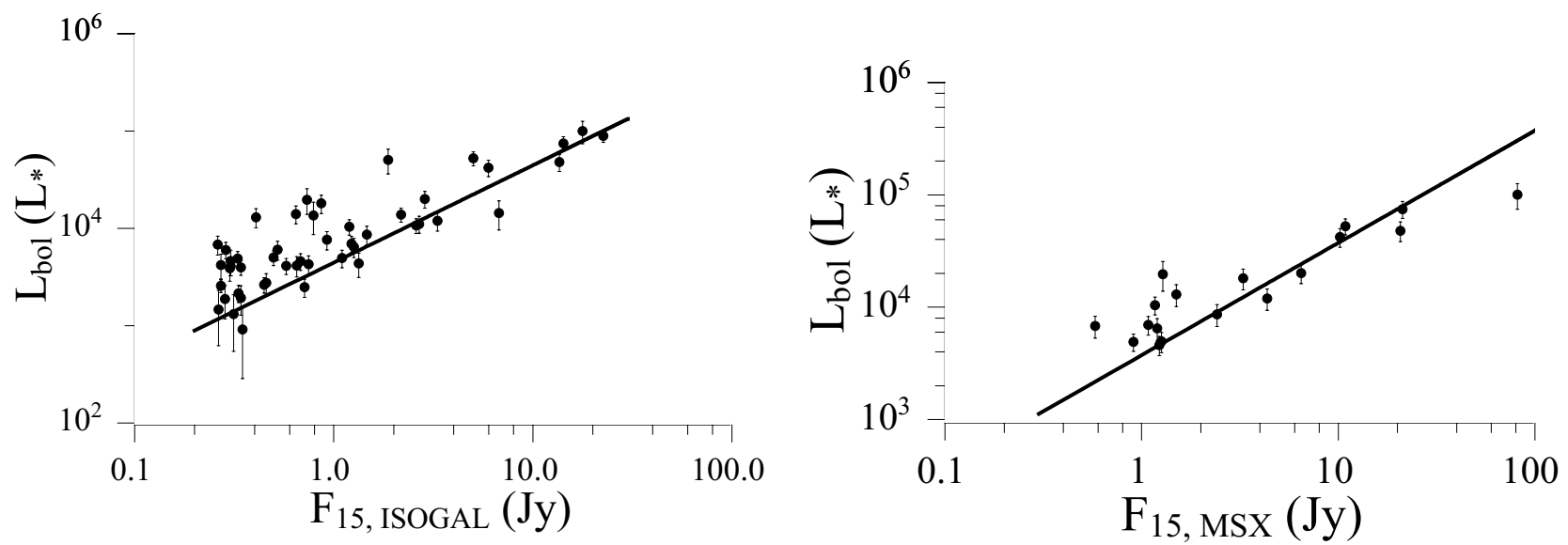

Fig. 9. Relation between the bolometric luminosity and the extinction-corrected ISOGAL (left) and MSX (right) flux densities at $15 \mu \mathrm{m}$ for the YOs in our test sample. The error bars correspond to the standard deviations of the bolometric luminosity values. The slopes of the fitted lines are $(4447 \pm 106) L_{\odot} / \mathrm{Jy}($ left $)$ and $(3736 \pm 209) L_{\odot} / \mathrm{Jy}($ right $)$, respectively.

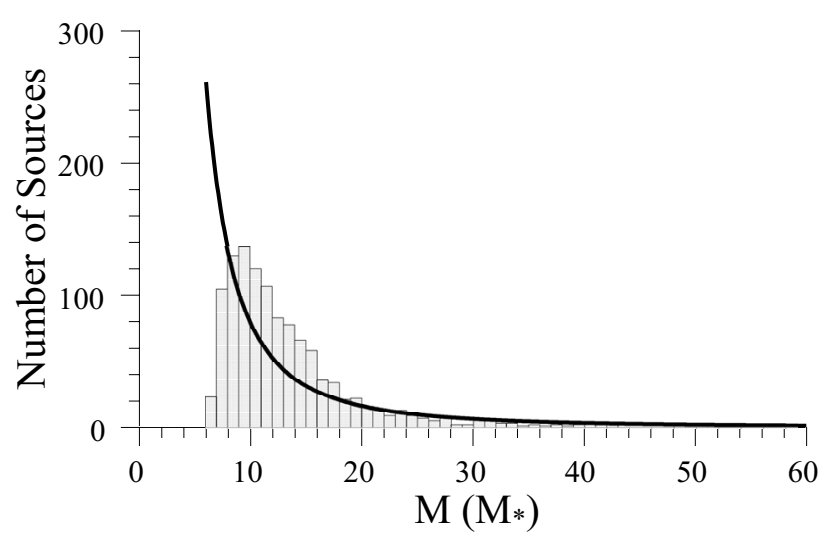

Fig. 10. Distribution of the source masses in our CMZ sample. The line represents the Kroupa IMF $\xi(M)=\xi_{0,1}(M) \cdot M^{-2.3}$. The histogram clearly shows that low-mass and very massive objects are missing in our CMZ sample due to our selection criterion [15] $<5.25$ mag and saturation effects, respectively.

of the mass distribution in the CMZ, we extrapolate the IMF to lower masses, following (Kroupa 2001)

$$
\begin{aligned}
& \xi(M)=\xi_{0,1}(M) M^{-2.3} \text { for } 0.5 M_{\odot} \leq M \leq 120 M_{\odot}, \\
& \xi(M)=\xi_{0,2}(M) M^{-1.3} \text { for } 0.08 M_{\odot} \leq M \leq 0.5 M_{\odot},
\end{aligned}
$$

and

$\xi(M)=\xi_{0,3}(M) M^{-0.3}$ for $0.01 M_{\odot} \leq M \leq 0.08 M_{\odot}$.

We fitted the histogram with a curve of the form $y=\xi_{0,1}(\mathrm{M}) \cdot \mathrm{x}^{-2.3}$ and obtained $\xi_{0,1}(M)=16019 M_{\odot}^{1.3}$ (see Fig. 10). In order to obtain a continous IMF, we derived $\xi_{0,2}(M)$ from $\xi_{0,1}(M)$ at $M=0.5 M_{\odot}$ and $\xi_{0,3}(M)$ from $\xi_{0,2}(M)$ at $M=0.08 M_{\odot}$, obtaining $\xi_{0,2}(M)=32038 M_{\odot}^{0.3}$ and $\xi_{0,3}(M)=400475 M_{\odot}^{-0.7}$. The total mass of YOs in the CMZ can then be derived with $M_{\mathrm{tot}}=\int M \xi(M) d M$. In the mass range $0.01-120 M_{\odot}$, the total mass is $\sim 77000 M_{\odot}$.

The histogram can be roughly divided into three mass ranges: $0-8 M_{\odot}, 8-20 M_{\odot}$, and $20-60 M_{\odot}$. As explained above, low-mass objects are missing in the lower mass range due to our selection criterion [15] $<5.25 \mathrm{mag}$. In the second mass range, the number of sources is underestimated by the fitted IMF by roughly $35 \%$ compared to the number of sources in our CMZ sample. Confusion due to the high population in the CMZ, saturation effects of the detectors, as well as rarity limit the detection of very luminous, hence massive, sources, which would explain the lack of objects in the upper mass range. As explained in Part I of this publication, we expect a contamination of our ISOGAL-CMZ sample with LEOs with a rate of $25 \%-35 \%$. If we assume that the MSX-CMZ sample is also contaminated with more evolved objects with a similar rate, we estimate the total contamination rate of our CMZ sample to be roughly $30 \%$. Thus, we expect to have $\sim 30 \%$ more sources in our CMZ sample over the whole mass range than predicted by the fitted IMF. This effect is not noticeable in the first and third mass ranges due to the number of missing objects. However, in the second mass range the larger number of sources in our sample compared to the number of sources predicted by the fitted IMF can be explained by the contamination of our sample with LEOs if we assume that our sample is complete in this mass range.

\subsubsection{Stellar clusters and the Sagittarius star-forming regions in the $\mathrm{CMZ}$}

Several regions in the CMZ are known as active star-forming sites such as the Sagittarius star-forming regions (Sgr B2+B1, Sgr C, Sgr D, Sgr E) and the three massive stellar clusters that are close to the Galactic center (Arches, Quintuplet, Central cluster). To obtain an estimate of the number of sources in our CMZ sample that are located in these active star-forming regions and therefore the influence of these sites on the star formation history in the CMZ, we counted the number of ISOGAL sources and MSX sources without an ISOGAL counterpart within the area of each of these star-forming regions. In Sgr B2 and B1 we found 35 ISOGAL and 32 MSX sources with a total mass of $930 M_{\odot}$. We counted 6 ISOGAL and 6 MSX sources in Sgr C with a total mass of $201 M_{\odot}$. In Sgr D, we detected only one MSX source with a mass of $42 M_{\odot}$. Neither ISOGAL nor MSX sources were detected in both Sgr E and the three stellar clusters. These regions are probably more evolved than our CMZ sample sources since they are several million years old, and their young stars are no longer deeply embedded in their natal cloud, which 
makes them appear less red. Our sample thus traces more recent star formation activity.

\subsubsection{Average star formation rate}

To appear bright at midinfrared wavelengths, the sources in our CMZ sample still have to be deeply embedded in the dust of their birth clouds. Following Wood \& Churchwell (1989), this phase lasts about $10 \%$ of the lifetime of an O-type or early B-type star, which corresponds roughly to $\sim 1$ Myr for a typical B0 star. Assuming that all objects in our CMZ sample have formed over an average time span of $1 \mathrm{Myr}$, we can convert the total mass of YOs $\left(\sim 77000 M_{\odot}\right)$ to an average star formation rate of $0.08 M_{\odot} \mathrm{yr}^{-1}$.

Previous studies (Crocker et al. 2011; Yusef-Zadeh et al. 2009) published a range of values for the star formation rate in the CMZ from $0.08-0.15 M_{\odot} \mathrm{yr}^{-1}$. An et al. (2011) make a rough estimate of the star formation rate based on the sources they have in common with the Yusef-Zadeh et al. source sample, deriving a value of $0.07 M_{\odot} \mathrm{yr}^{-1}$. Although our calculations are based on a number of simplifying assumptions (e.g. linear relation between $F_{15}$ and $L_{\text {bol }}$, transformation of the bolometric luminosities into masses of single ZAMS stars, etc.), we can state that our result is consistent with other independent studies of the star formation rate.

\section{Summary}

The goal of this study was to test and revise ISOGAL selection criteria on a test sample of 68 unknown ISOGAL sources and to apply these criteria to all ISOGAL point sources in the CMZ in order to find YOs and derive the average star formation rate over the past 1 Myr. We obtained Spitzer/IRS observations for a test sample of 68 ISOGAL sources and assembled spectra in the wavelength range 5-38 $\mu \mathrm{m}$ for 57 of these sources. Depending on the detection of PAH or forbidden fine structure line emission and the slope of the spectrum, we classified the sources as YOs or LEOs.

After revising the ISOGAL selection criteria, which are based on the ISOGAL [7]-[15] color and the spatial extent parameter $\sigma_{15}$, we applied them to all ISOGAL sources in the $\mathrm{CMZ}\left( \pm 1.5^{\circ} \times \pm 0.5^{\circ}\right.$ around the Galactic center $)$, selecting 485 sources. Furthermore, we added $656 \mathrm{MSX}$ sources in the CMZ that fulfilled the criterion $F_{\mathrm{E}} / F_{\mathrm{D}} \geq 2\left(F_{\mathrm{D}}, F_{\mathrm{E}}\right.$ flux densities in band $D(15 \mu \mathrm{m})$ and $\mathrm{E}(21 \mu \mathrm{m}))$.

After fitting the SEDs of the 47 YOs in our test sample, we obtained bolometric luminosity values and plotted them separately against the extinction-corrected ISOGAL and MSX flux densities at $15 \mu \mathrm{m}$ of the sources. We obtained $\frac{L_{\text {bol }}}{F_{15}}$ conversion factors and applied them to our CMZ sample. Assuming that the bolometric luminosity of each source corresponds to the luminosity of a ZAMS star, we calculated the mass for each source. The distribution of the masses roughly follows a Salpeter or Kroupa IMF, but low-mass and very massive objects are missing from the CMZ sample. The total mass of YOs in the CMZ $\left(\sim 77000 M_{\odot}\right)$ was calculated from a Kroupa IMF between 0.01 and $120 M_{\odot}$. Since the detection as bright midinfrared sources requires that the sources are still deeply embedded in their dust cocoon, the sources still have to be very young. Assuming that this total mass in YOs has formed over the past $1 \mathrm{Myr}$, we obtained a star formation rate of $0.08 M_{\odot} \mathrm{yr}^{-1}$ for the $\mathrm{CMZ}$, that is consistent with previous studies.

\section{References}

An, D., Ramírez, S. V., Sellgren, K., et al. 2011, ApJ, 736, 133

Bhattacharya, A., Joardar, S., \& Bhattacharya, R. 2008, A\&A (Infinity Science Press LLC)

Crocker, R. M., Jones, D. I., Aharonian, F., et al. 2011, MNRAS, 413, 763

Eddington, A. S. 1926, The Internal Constitution of the Stars (Cambridge University Press)

Egan, M. P., Price, S. D., Kraemer, K. E., et al. 2003, VizieR Online Data Catalog, 5114

Felli, M., Testi, L., Schuller, F., \& Omont, A. 2002, A\&A, 392, 971

Fiolet, N., Omont, A., Lagache, G., et al. 2010, A\&A, 524, A33

Gerakines, P. A., Schutte, W. A., Greenberg, J. M., \& van Dishoeck, E. F. 1995, A\&A, 296, 810

Glass, I. S., Matsumoto, S., Carter, B. S., \& Sekiguchi, K. 2001, MNRAS, 321, 77

Griffiths, S. C., Hicks, R. B., \& Milone, E. F. 1988, JRASC, 82, 1

Güsten, R. 1989, in The Center of the Galaxy, ed. M. Morris, IAU Symp., 136, 89

Houck, J. R., Roellig, T. L., Van Cleve, J., et al. 2004, in SPIE Conf. Ser. 5487, ed. J. C. Mather, 62

Jiang, B. W., Gao, J., Omont, A., Schuller, F., \& Simon, G. 2005, VizieR Online Data Catalog, 344, 60551

Kaufman, M. J., Wolfire, M. G., \& Hollenbach, D. J. 2006, ApJ, 644, 283

Kroupa, P. 2001, MNRAS, 322, 231

Lumsden, S. L., Hoare, M. G., Oudmaijer, R. D., \& Richards, D. 2002, MNRAS, 336,621

Molster, F. J., Waters, L. B. F. M., \& Tielens, A. G. G. M. 2002, A\&A, 382, 222

Morris, M., \& Serabyn, E. 1996, ARA\&A, 34, 645

Nishiyama, S., Nagata, T., Tamura, M., et al. 2008, ApJ, 680, 1174

Omont, A., Gilmore, G. F., Alard, C., et al. 2003, A\&A, 403, 975

Price, S. D., Egan, M. P., Carey, S. J., Mizuno, D. R., \& Kuchar, T. A. 2001, AJ, 121,2819

Quirico, E., Raynal, P., Borg, J., et al. 2000, in Lunar and Planetary Inst. Technical Report, Lunar and Planetary Institute Science Conference Abstracts, 31, 1260

Reid, M. J., Menten, K. M., Zheng, X. W., Brunthaler, A., \& Xu, Y. 2009, ApJ, 705,1548

Robitaille, T. P., Whitney, B. A., Indebetouw, R., Wood, K., \& Denzmore, P. 2006, ApJS, 167, 256

Robitaille, T. P., Whitney, B. A., Indebetouw, R., \& Wood, K. 2007, ApJS, 169, 328

Rodríguez-Fernández, N. J., Martín-Pintado, J., Fuente, A., \& Wilson, T. L. 2004, A\&A, 427, 217

Salpeter, E. E. 1955, ApJ, 121, 161

Schuller, F., Ganesh, S., Messineo, M., et al. 2003, A\&A, 403, 955

Schuller, F., Omont, A., Glass, I. S., et al. 2006, A\&A, 453, 535

Schultheis, M., Ganesh, S., Simon, G., et al. 1999, A\&A, 349, L69

Schultheis, M., Lançon, A., Omont, A., Schuller, F., \& Ojha, D. K. 2003, A\&A, 405,531

Schultheis, M., Sellgren, K., Ramírez, S., et al. 2009, A\&A, 495, 157

Schutte, W. A., Greenberg, J. M., van Dishoeck, E. F., et al. 1998, Astrophys. Space Sci., 255, 61

Sjouwerman, L. O., van Langevelde, H. J., Winnberg, A., \& Habing, H. J. 1998, A\&AS, 128, 35

Werner, M. W., Roellig, T. L., Low, F. J., et al. 2004, ApJS, 154, 1

Wood, D. O. S., \& Churchwell, E. 1989, ApJ, 340, 265

Yusef-Zadeh, F., Hewitt, J. W., Arendt, R. G., et al. 2009, ApJ, 702, 178 
K. Immer et al.: Recent star formation in the inner Galactic bulge. II.
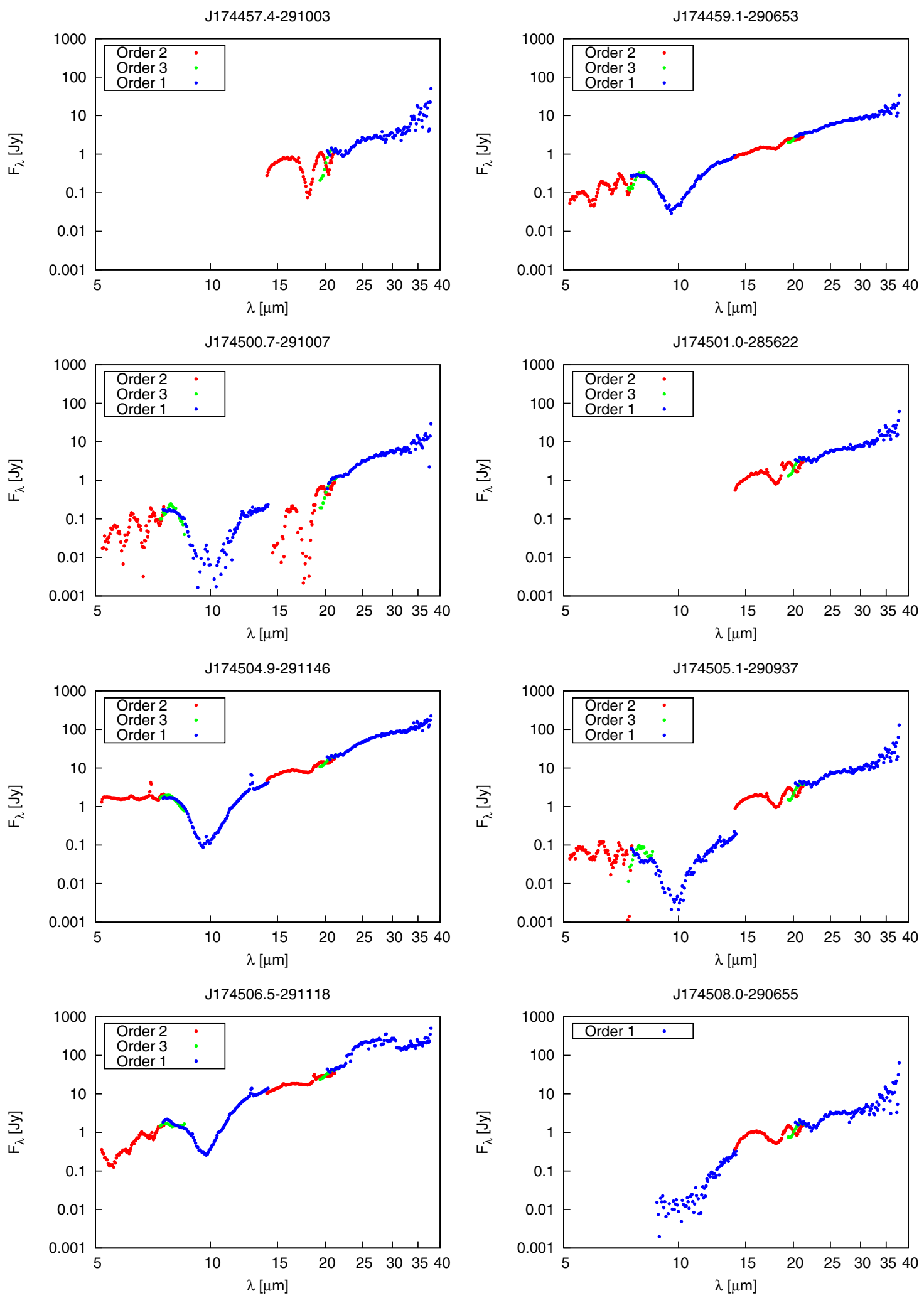

Fig. 3. Background subtracted spectra. The data of the different subslits are indicated by the three different colors (green: SL2 or LL2; blue: SL3 or LL3; red: SL1 or LL1). 

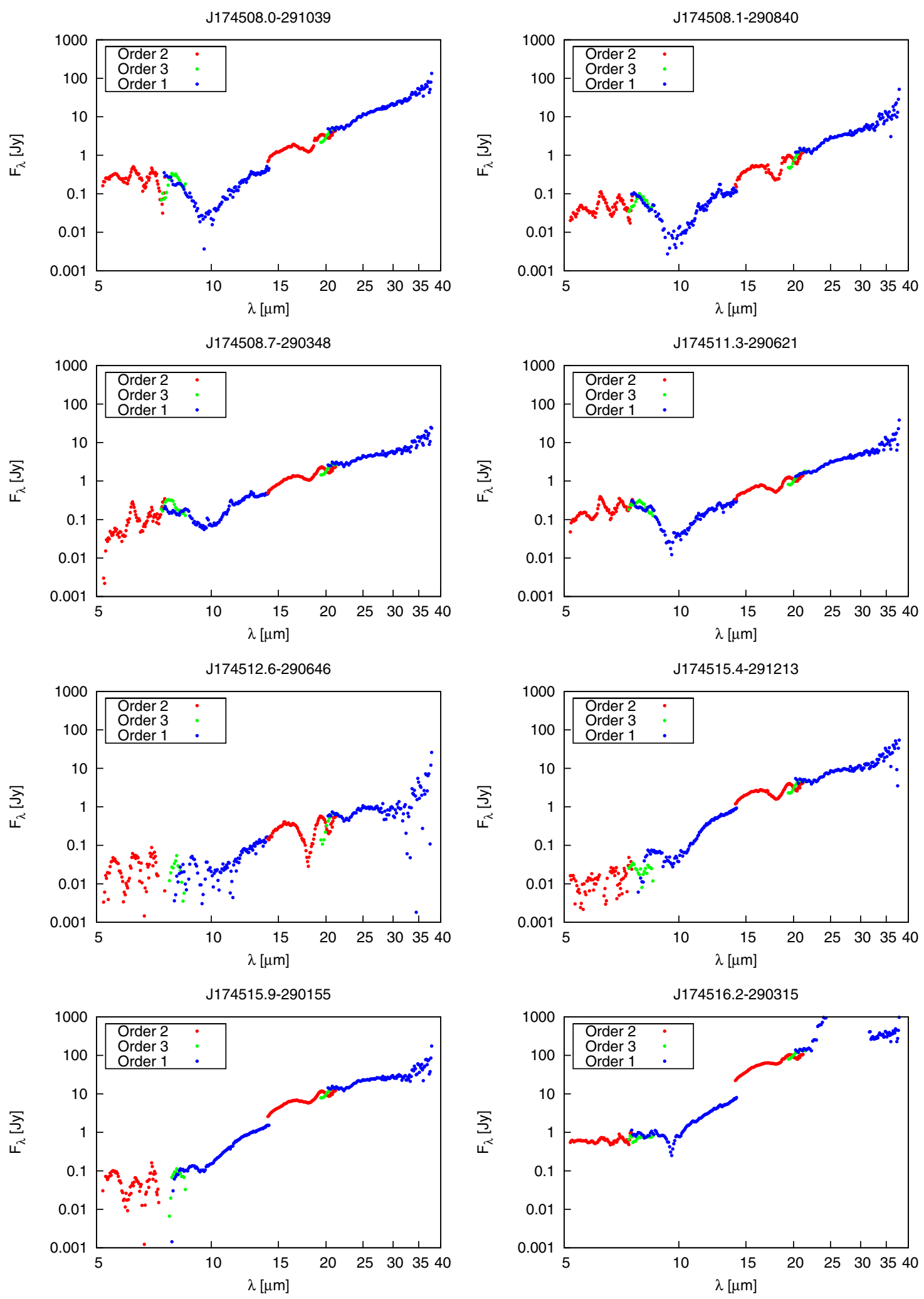

Fig. 3. continued. 
K. Immer et al.: Recent star formation in the inner Galactic bulge. II.
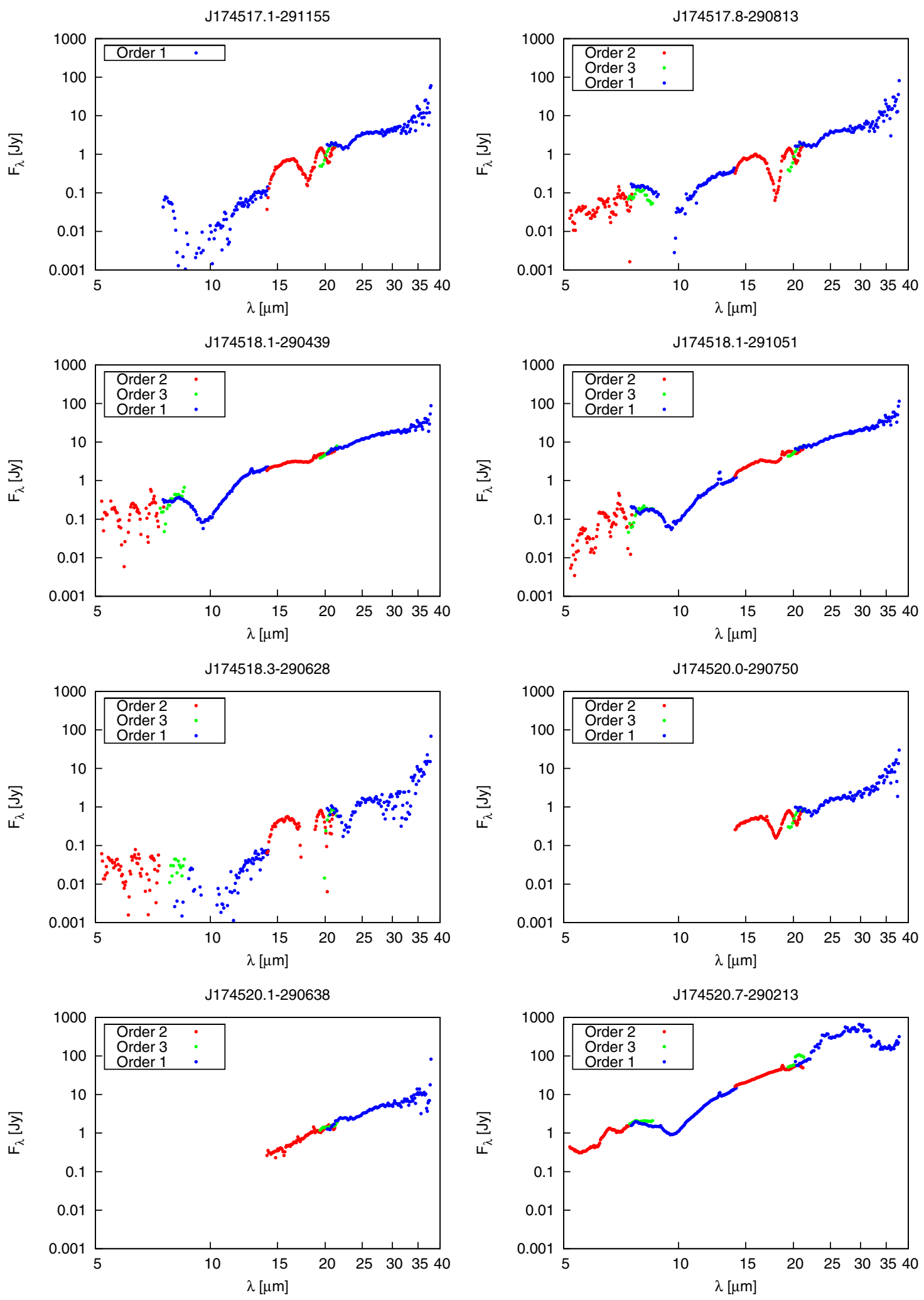

Fig. 3. continued. 

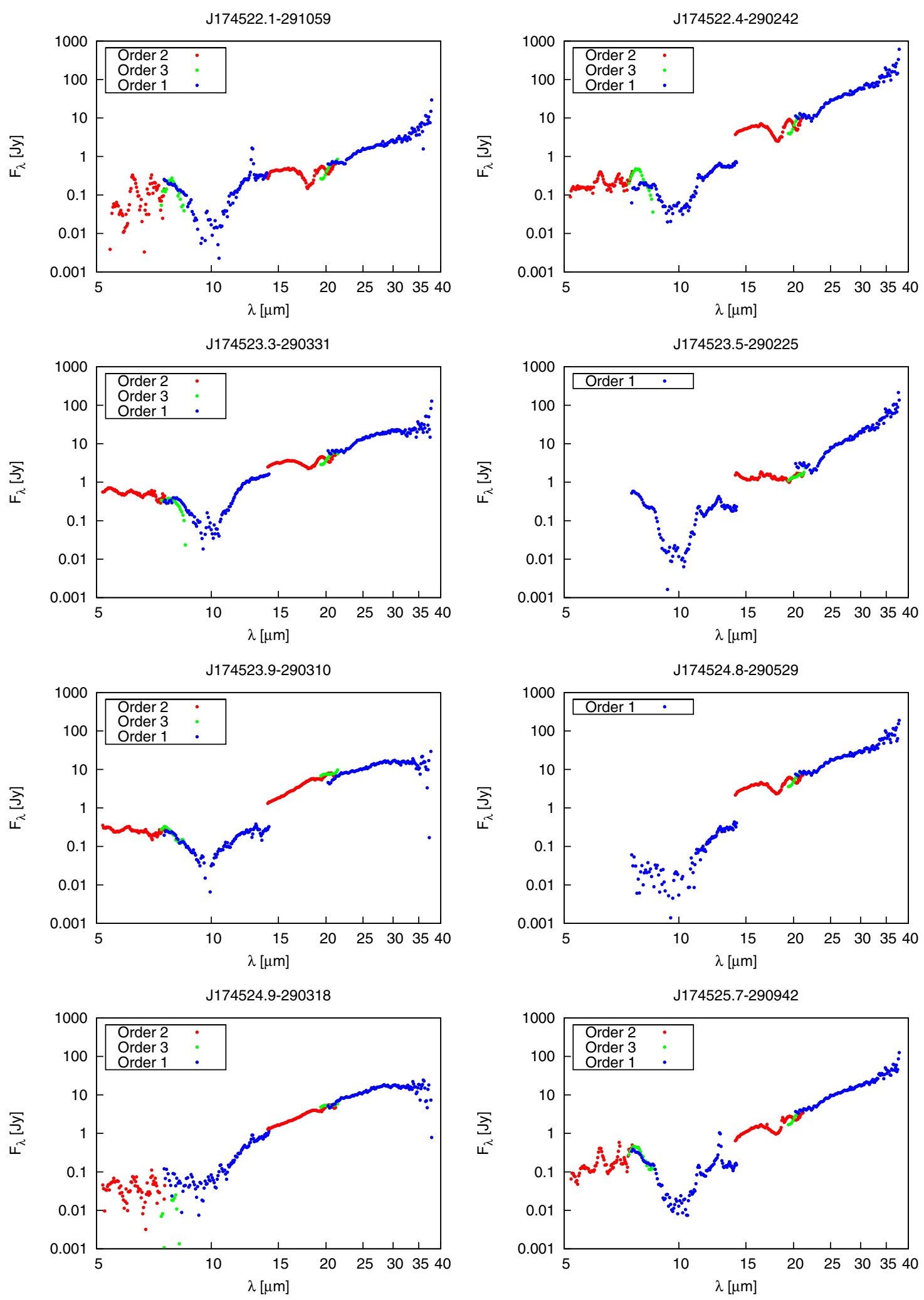

Fig. 3. continued. 
K. Immer et al.: Recent star formation in the inner Galactic bulge. II.
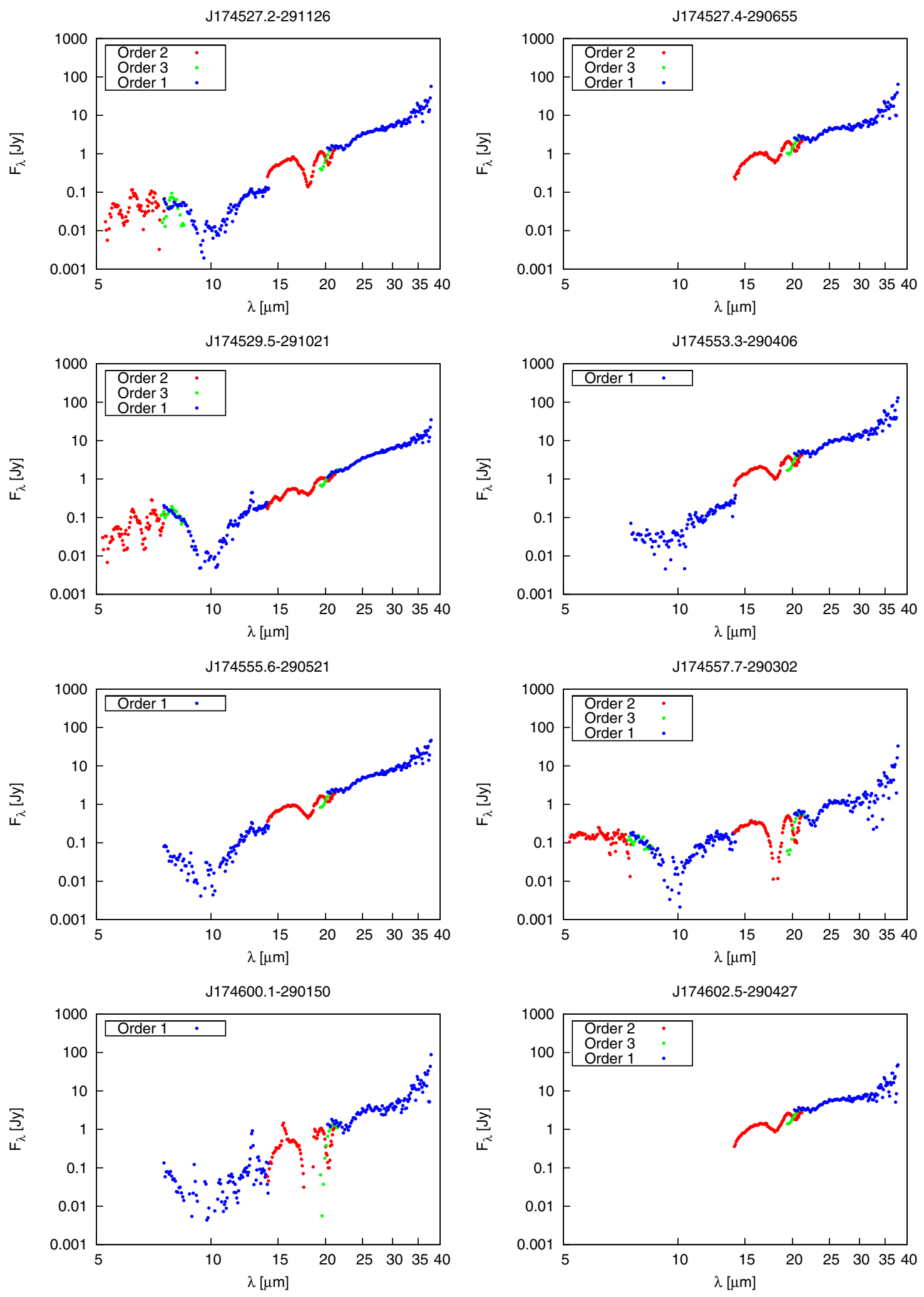

Fig. 3. continued. 

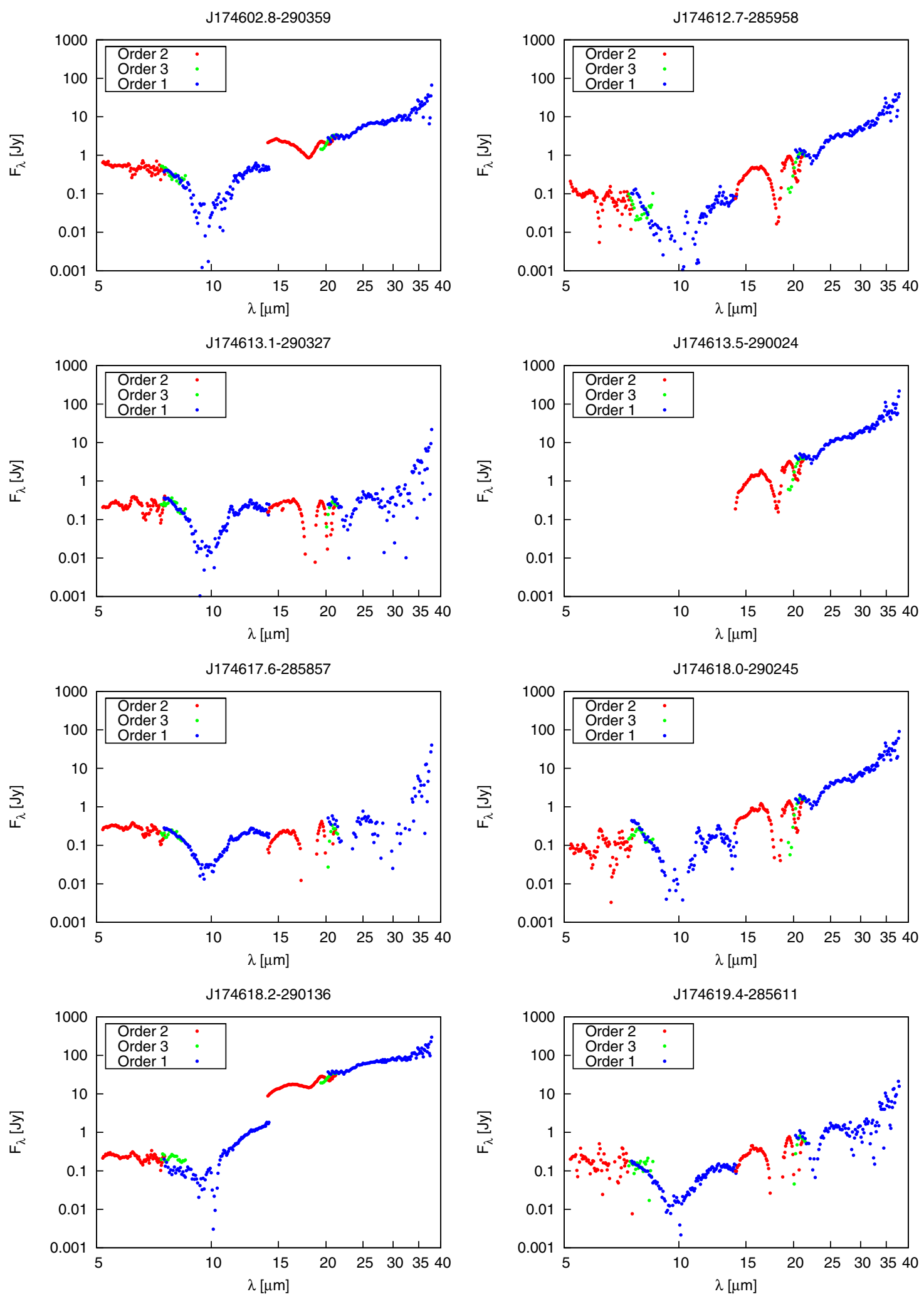

Fig. 3. continued. 
K. Immer et al.: Recent star formation in the inner Galactic bulge. II.
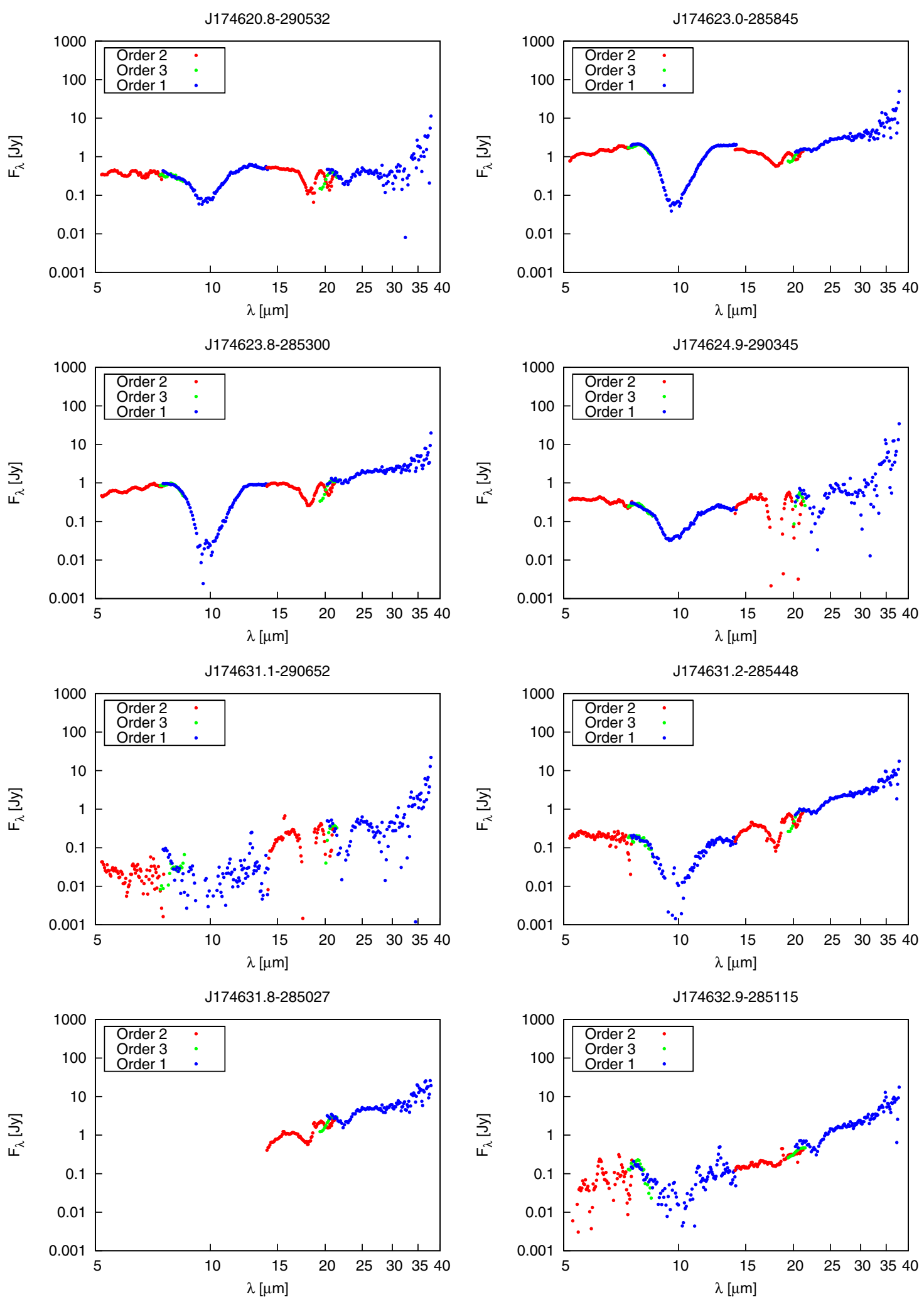

Fig. 3. continued. 